\title{
A Theory of Auctions with Endogenous Valuations
}

\author{
Alex Gershkov, Benny Moldovanu, Philipp Strack and Mengxi Zhang*
}

July 3,2020

\begin{abstract}
We derive the revenue maximizing allocation of $m$ units among $n$ symmetric agents who have unit demand, and who take costly actions that influence their values before participating in the mechanism. The allocation problem with costly actions can be represented by a reduced form model where agents have convex, non-expected utility preferences over the interim probability of receiving an object. Both the uniform $m+1$ price auction and the discriminatory pay-your-bid auction with reserve price constitute symmetric revenue maximizing mechanisms. Contrasting the case with exogenous valuations, the optimal reserve price reacts to both demand and supply. We also identify a condition under which the optimal mechanism is indeed symmetric, and illustrate the structure of the optimal asymmetric mechanism when the condition fails. The main tool in our analysis is an integral inequality, due to Fan and Lorentz (1954), involving majorization, super-modularity and convexity.
\end{abstract}

${ }^{*}$ The present paper combines material from an earlier paper (with the same name) by the first three authors and from Zhang (2018). We are grateful to Emir Kamenica and to 4 anonymous referees for their editorial comments. We wish to thank David Dillenberger, Philippe Jehiel, Daniel Kraehmer, Edi Karni, Andreas Kleiner, Bart Lipman, Xianwen Shi, Andrzej Skrzypacz and Rakesh Vohra for helpful comments. Moldovanu acknowledges financial support from the German Science Foundation via the Hausdorff Center for Mathematics, the CRC TR-224 (Project B01) and Germany's Excellence Strategy - EXC 2126/1390838866. Gershkov wishes to thank the Israel Science Foundation for financial support. Zhang wishes to acknowledge financial support from the German Science Foundation via the Hausdorff Center for Mathematics and CRC TR-224 (Project B01). Gershkov: Department of Economics and the Federmann Center for the Study of Rationality, The Hebrew University of Jerusalem, and School of Economics, University of Surrey, alexg@huji.ac.il; Moldovanu: Department of Economics, University of Bonn, mold@uni-bonn.de; Strack: Department of Economics, Yale University, New Haven, philipp.strack@yale.edu; Zhang: Department of Economics, University of Bonn, mzhang@uni-bonn.de. 


\section{Introduction}

We derive the revenue maximizing mechanism in a multi-unit allocation problem where agents, prior to interacting with the mechanism, undertake costly actions that are unobservable to the designer, and that influence their valuations. ${ }^{1}$ All other model's features are standard, and correspond to the symmetric, private independent values model with quasilinear utility. The results immediately translate to procurement setting where the designer acts as the buyer. ${ }^{2}$

Because of the ex-ante investments, the agents' valuations become endogenous to the mechanism. For example, if the action is a costly investment that increases an agent's value for the good, this agent will only undertake the investment if she believes that her probability of receiving an object in the mechanism is sufficiently high. Hence, in contrast to the standard environment where values are exogenous, a mechanism also needs to provide here the right incentives for individual investments, as it indirectly affects revenue by changing the distribution of valuations.

A revenue-maximizing seller can provide stronger ex-ante investment incentives by increasing the probability of allocating units, but is constrained by the limited supply and by the usual monopolistic supply reduction incentives. The revenue maximizing mechanism must finely balance these conflicting forces. The revenue-maximizing mechanism for any fixed profile of investment strategies can be found by standard methods following Myerson's (1981) celebrated analysis. But, such a mechanism will not be optimal because its derivation ignores the indirect effect of the mechanism on the distribution of values. To directly solve for the optimal mechanism that is selected before agents invest, one would need to:

1. Characterize the endogenous distribution of valuations generated by the investments induced by each feasible mechanism.

2. Derive the expected revenue for each feasible mechanism (taking into account the endogenous distribution of values it generates) and maximize over feasible mechanisms.

Both steps pose major challenges that have hindered a solution so far: an agent's investment in a given mechanism depends on the anticipated bids and thus the distribution of

\footnotetext{
${ }^{1}$ Tan (1992) documents an example where two groups of military contractors spent over $\$ 600$ million each, prior to competitive bidding.

${ }^{2} \mathrm{~A}$ variety of settings form special cases of our maximization framework and we offer illustrations to auctions where the endogenous values are influenced either additively or multiplicatively by costly actions, and to auctions with entry costs.
} 
other agents' investments - the overall distribution of investments must solve a fixed point problem. Moreover, the distribution of values and hence of virtual values is also endogenously determined by the mechanism.

The principal innovation of the paper is a solution via an approach that avoids the above complex steps by combining: 1) a reduced-form modeling where agents have non-expected utility preferences that are convex in the interim probability of obtaining a unit, and 2) optimization based on the Fan-Lorentz (1954) integral inequality.

It is well known from the decision-theoretic literature (see, for example, Kreps and Porteus 1979 and Machina (1984)) that preferences induced by actions that are taken prior to the resolution of uncertainty become non-linear and convex in probabilities - this is, precisely, the insight we exploit here by solving, instead of the original auction problem with investments, a reduced-form auction problem without investments, but with convex non-expected utility preferences. ${ }^{3}$

The reduced form model presents its own challenges: the main technical difficulty is the non-linearity of ex-ante valuations in the allocation probability, which implies that the revenue maximization exercise cannot be performed "realization by realization". The direct subject of maximization becomes the interim probability of obtaining an unit - the "reduced form auction" in the language of Maskin and Riley (1984), Matthews (1984) and Border (1991). Therefore, besides the standard monotonicity requirement stemming from incentive compatibility, the most complex constraint is the feasibility constraint, derived for multiunit auctions by Che, Kim and Mierendorff (2013). To solve the optimization problem we combine insights gained from majorization theory (see Hardy, Littlewood and Polya 1929) with the elegant Fan and Lorentz (1954) integral inequality that determines the maximizer of a convex and super-modular objective subject to a majorization constraint.

We first focus on symmetric mechanisms: under a super-modularity and convexity condition on the preferences, the optimization exercise over direct mechanisms yields an interim probability assignment such that units are allocated to the agents with the highest types, conditional on these exceeding a critical cutoff. ${ }^{4}$ We also derive the bidding equilibria of the uniform $(m+1)$ - price auction and of the discriminatory pay-your-bid auction with a reserve price (where $m$ is the number of supplied units), and show that these auctions, aug-

\footnotetext{
${ }^{3}$ Non-linear preferences in probabilities have also appear in Kahneman and Tversky's (1979) Prospect Theory and Quiggin (1982). Consequences of multi-agent equilibrium behavior were first analyzed by Crawford (1990).

${ }^{4}$ The present optimization approach throws a new light also on the classical results obtained in the linear case, masterfully analyzed by Myerson (1981) and by Riley and Samuelson (1981) - their setting with exogenous valuations is a special case of our present framework.
} 
mented by a reserve price, implement the revenue maximizing allocation among symmetric mechanisms.

While in the setting with exogenous valuations the optimal reserve price coincides with the optimal cutoff, and neither is responsive to changes in demand nor supply, here we find that reserve price and cut-off type are distinct objects, and often display comparative statics in opposite directions: Under the same general conditions used for the maximization exercise, the optimal cutoff increases in the number of agents, and decreases in the number of objects, and under some additional sufficient conditions, the optimal reserve price decreases in the number of agents and increases in the number of objects.

For the one-object and two-bidder case, we also provide a sufficient (and almost necessary) condition under which the optimal mechanism is indeed symmetric. As is well known from Myerson's analysis, in symmetric frameworks a discriminatory treatment decreases the payment that can be extracted from agents with an exogenously given distribution of values. On the other hand, by giving a bidder an "unfair" advantage over competitors, the seller can increase this particular bidder's investment incentives. Roughly speaking, our condition ensures that the first effect dominates. The technical argument involves a combination of the Fan-Lorentz insight, Young's inequality, and a variational principle.

In addition, we provide an illustrative example where we uncover the structure of the optimal asymmetric mechanism when the above condition fails: this mechanism combines, or, in the extreme cases replaces, the auction by a series of posted-price offers with favoritism.

Finally, we also note that, if the optimal mechanism is asymmetric, the seller can increase revenue in a standard, symmetric auction by restricting the entry of bidders ${ }^{5}$. This ensures that each bidder has a reasonably high chance of winning, and hence a high enough incentive to invest. In particular, the classic result of Bulow and Klemperer (1996) need not to hold in our environment.

The paper is organized as follows: At the end of the present section we briefly survey the relevant literature. In Section 2 we describe the auction model. In Section 3 we formulate the revenue maximization problem and the main tools towards its solution. In Section 4 we derive the symmetric revenue maximizing allocation in the "regular" case, we offer a condition when this is the overall optimal mechanism, and discuss the optimal asymmetric mechanism when this condition fails. We also illustrate how ex-ante restricting entry can be beneficial. The focus of Section 5 is on standard auction formats that implement the

\footnotetext{
${ }^{5}$ In projects where investments are massive, only two bidders are allowed. See Fullerton and McAfee (2009) and Che and Gale (2003) for examples in procurement.
} 
optimal allocation, and we explain how the optimal reserve price is affected by demand and supply. This section concludes by showing how the uniform price auction can be also used to implement the welfare maximizing allocation. In Section 6 we briefly investigate the welfare maximizing mechanism and compare it with the revenue maximizing one. Section 7 concludes. All proofs and some additional derivations are contained in two Appendices.

\subsection{Literature Review}

Single-object, standard auctions with non-expected utility preferences were analyzed by, among others, Karni and Safra (1989) and by Neilson (1984).

There is a substantial literature on auctions with investments, often formulated in the dual procurement setting where the designer acts as a buyer. In that literature, the mechanism is taken there as given - often a first- or second-price auction - and an optimal mechanism-design analysis is not performed. King et al. (1992) consider completely informed bidders whose types depend on investments made prior to the auction, and show that the second-price auction leads to efficient investments (only one buyer invests), while the first-price auction (i.e, its mixed-strategy symmetric equilibrium) yields a higher revenue. Rogerson (1992) and Hatfield et al. (2018) relate the ex-post efficient allocation to strategy-proofness and ex-ante incentives to invest.

Piccione and Tan (1996), Tan (1992), and Jeitschko and Wolfstetter (2000) analyze models where the effect of investments is stochastic. The incentives of the designer to create asymmetries among otherwise ex-ante identical bidders in procurement with observable investments are discussed by Bag (1997) and by Arozamena and Cantillon (2004).

Another related literature stream studies auctions with entry cost: entry can be seen as a binary investment decision with a fixed cost, and the optimal auction derivation is then more amenable to standard analysis. The settings of Celik and Yilankaya (2009) and Menezes and Monteiro (2000), for example, are special cases of our model because the entry decision is made by privately informed agents.

\section{Auctions with Ex-Ante Investments}

There are $m \geq 1$ identical and indivisible objects, and $n \geq m$ ex-ante symmetric agents.

Each agent $i \in\{1, \ldots, n\}=N$ has a type $\theta_{i} \in \Theta=[\underline{\theta}, \bar{\theta}] \subseteq \mathbb{R}_{+}$that is her private information, and demands at most one object. Types are distributed I.I.D. according to a distribution $F: \Theta \rightarrow[0,1]$, with positive density $f>0$. 


\subsection{Actions and Preferences}

Before participating in the mechanism, each agent $i$ takes an action $a_{i}$ from a compact set $A \subset \mathbb{R}$. This action is private information to the agent (and therefore unobservable to the designer). Depending on her type $\theta_{i}$, agent $i$ has preferences over her own action $a_{i} \in A$, her own allocation $x_{i} \in[0,1]$ and her own monetary transfer $y_{i}$. We assume that these are standard expected utility preferences, quasi-linear in the monetary transfer and separable in the cost of an action $c\left(a_{i}\right) \geq 0$. The preference can be thus represented by a utility function of the form:

$$
x_{i} v\left(a_{i}, \theta_{i}\right)-y_{i}-c\left(a_{i}\right),
$$

where the value for a unit of the good, $v\left(a_{i}, \theta_{i}\right) \in \mathbb{R}_{+}$, is assumed to be increasing in her type $\theta_{i}$ and in her action $a_{i}$, super-modular and non-negative. ${ }^{6}$ In addition, we assume that $v$ is differentiable and that its partial derivative with respect to $\theta$ is bounded. Furthermore, we assume that there exists a costless action $0 \in A$ such that $c(0)=0{ }^{7}$

Remark. The agent's action may also have an effect outside the mechanism, e.g., an investment in a cost reducing technology produces additional benefits in other areas of operation. We can include this in our analysis by having $v\left(a_{i}, \theta_{i}\right)$ measure the effect of the costly action on the valuation inside the mechanism, and by having $c\left(a_{i}\right)$ measure the cost, net of the benefits, outside the mechanism.

\subsection{The Dual Procurement Model}

The auction model is equivalent to a model of procurement where the designer is a buyer and where seller $i$ incurs a cost of $\tilde{v}\left(a_{i}, \theta_{i}\right)$ if she produces an unit. Higher investment levels, or higher types lead to lower cost, and the cost $\tilde{v}$ is sub-modular: higher types reduce their cost more by investing.

Without loss of generality, assume that the buyer pays every winning seller the cost incurred by the highest cost seller type under no investment, $\tilde{v}(0, \underline{\theta})$, and asks the seller to pay back an amount $t_{i}$. Seller $i$ 's utility is thus given by

$$
-x_{i} \tilde{v}\left(a_{i}, \theta_{i}\right)+x_{i} \tilde{v}(0, \underline{\theta})-t_{i}-c\left(a_{i}\right)=x_{i} v\left(a_{i}, \theta_{i}\right)-t_{i}-c\left(a_{i}\right)
$$

\footnotetext{
${ }^{6}$ The assumption that the value is non-negative corresponds to free disposal.

${ }^{7}$ This is a normalization: we can always subtract $\min _{a \in A} c(a)$ from the agent's utility without changing her preferences over actions, allocations, or transfers.
} 
where we define $v\left(a_{i}, \theta_{i}\right)=\tilde{v}(0, \underline{\theta})-\tilde{v}\left(a_{i}, \theta_{i}\right)$. Then, the value function $v$ satisfies all assumptions made above, and therefore the optimal mechanism for procurement can be determined from the optimal mechanism in the auction problem, and vice-versa.

\subsection{Timing and Discussion}

The timing is as follows (c.f. Figure 1):

1. Each agent privately observes her type;

2. The designer (i.e., seller in the auction interpretation) commits to a mechanism;

3. Each agent decides whether to participate in the mechanism, and privately chooses an action; the taken action is not observable by other agents nor by the designer.

4. Each agent sends a message to the mechanism;

5. Depending on the sent messages, an allocation and transfers are realized.

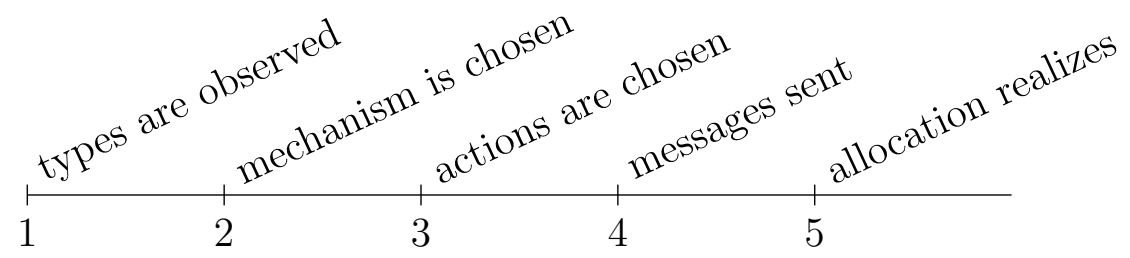

Figure 1: Timing of the game.

This is the standard timing in the literature on procurement auctions with costly investments to reduce $\operatorname{cost}^{8}$. In particular, it restricts attention to commonly used mechanisms where messages are exchanged between the agents and the seller only after actions are chosen. Since the seller indirectly cares about efficiency (because it increases the pie from which revenue can be extracted) and since it is inefficient to have more than $m$ agents pay the investment cost, the seller would like to coordinate investments, e.g., by adding a round of communication prior to investment. Alternatively, the seller could run the auction before

\footnotetext{
${ }^{8}$ Building a prototype for a battleship or a jet fighter, say, requires an adjustment of existing technologies, and may lead to a future cost reduction, which is equivalent to an investment in the object's value in the standard auction setup.
} 
investments are made, and then allow winners to make investments before ultimately delivering the objects and collecting payments. While theoretically beneficial, such a delay is often not practical when the involved periods of time are substantial (see the discussion of ex-ante investments in the context of the recent FCC incentive auction in Milgrom (2017)). ${ }^{9}$ For example, the government could hold an auction in 2020 for the right to start using new spectrum in, say, 2030. But then firms need to make large monetary commitments without knowing which technology will emerge from their investments, and the winners need not be those with the best future technology. Second, due to liquidity constraints, it may not be feasible to collect the full payment today, leading to possible renegotiation in the future. ${ }^{10}$

\subsection{Mechanisms}

A mechanism specifies a set of reports $R_{i}$ for each agent $i$, and a mapping from reports to an allocation and transfers:

$$
\begin{aligned}
& x: \prod_{i \in N} R_{i} \rightarrow X=[0,1]^{n} \\
& y: \prod_{i \in N} R_{i} \rightarrow \mathbb{R}^{n} .
\end{aligned}
$$

Given a mechanism $(x, y)$ each agent $i$ picks an optimal report $r_{i}$ and takes an optimal action. As agent $i$ can condition her action on the report she plans to send, her reporting problem is equivalent to

$$
\max _{r_{i} \in R_{i}}\left(\left\{\max _{a_{i} \in A} \mathbb{E}_{r_{-i}}\left[x_{i}(r)\right] v\left(a_{i}, \theta_{i}\right)-c\left(a_{i}\right)\right\}-\mathbb{E}_{r_{-i}}\left[y_{i}(r)\right]\right) .
$$

\subsection{Reduction to Convex Preferences}

We now show that an agent who takes an endogenous action obtains the same interim expected utility and sends the same report as an agent who takes no action, but has a

\footnotetext{
${ }^{9}$ For example, incumbent potential winners at the $20195 \mathrm{G}$ spectrum auction in Germany were required to operate at least $10005 \mathrm{G}$ basis stations each until 2022, and to offer speeds of at least 100Mbit/second on all major rail-and highways until 2024. Newcomers must cover 25\% of the population until 2023 and $50 \%$ until 2025.

${ }^{10}$ Stochastic investments and moral hazard - are not yet part of our current mode. But, it is of importance to understand the properties of mechanisms actually used in practic. A future, more complex, model may explicitly incorporate an optimization over the time structure.
} 
non-expected utility preference. Define

$$
p_{i}\left(r_{i}\right):=\mathbb{E}_{r_{-i}}\left[x_{i}(r)\right]
$$

to be the interim probability with which agent $i$ receives an object in a given mechanism. Let $h:[0,1] \times \Theta \rightarrow \mathbb{R}$ be the utility agent $i$ gets when she receives an unit with probability $p_{i}$ and takes the optimal action ${ }^{11}$

$$
h\left(p_{i}, \theta_{i}\right):=\max _{a_{i} \in A} p_{i} v\left(a_{i}, \theta_{i}\right)-c\left(a_{i}\right) .
$$

Then, the reporting problem of agent $i$ given in (1) is equivalent to the reduced form problem where agent $i$ has non-linear preferences over her allocation probability $p_{i}$

$$
\max _{r_{i} \in R_{i}} h\left(p_{i}\left(r_{i}\right), \theta_{i}\right)-\mathbb{E}_{r_{-i}}\left[y_{i}(r)\right]
$$

Lemma 1. $h\left(p_{i}, \theta_{i}\right)$ is convex in $p_{i}$, increasing in $\theta_{i}$ and $p_{i}$, super-modular in $\left(p_{i}, \theta_{i}\right)$ and satisfies $h\left(0, \theta_{i}\right)=0$ for all $\theta_{i} \in \Theta$.

The agent's incremental benefit from receiving the object with higher probability is nonnegative, and higher types derive higher utility from the object. Since $h$ is defined independently of the agent's action, the mechanism design problem where the agent chooses an action before participating in the mechanism is equivalent to the mechanism design problem where each agent has a convex valuation given by $h\left(p_{i}, \theta_{i}\right)$, but chooses no costly action.

\section{The Revenue Maximization Problem}

We first restrict attention to symmetric mechanisms that are invariant to permutations of the agents' names, and where every agent uses the same reporting and investment strategies.

Consider a symmetric, direct mechanisms where each agent reports his type $\theta_{i}$, and where the mechanism specifies allocations and monetary transfers to all agents, depending on the reported types. As $h\left(p_{i}, \theta_{i}\right)$ is super-modular, it is well-known that the incentive compatibility of a (symmetric) direct mechanism is equivalent to the standard monotonicity

\footnotetext{
${ }^{11}$ We assume that an optimal action always exists, which is satisfied for example if $\frac{\partial^{2} v}{\partial a^{2}} \leq 0$ for all $\theta_{i}$ and $\frac{\partial^{2} c}{\partial a^{2}}>0$
} 
of the equilibrium expected allocation ${ }^{12}$

$$
p\left(\theta_{i}\right):=\mathbb{E}_{\theta_{-i}}\left[x_{i}\left(\theta_{1}, \ldots, \theta_{n}\right)\right]
$$

together with an envelope condition determining the expected interim transfer (see for example Guesnerie and Laffont (1984), Corollary 2.1). The envelope condition yields the following standard revenue formula ${ }^{13}$ :

Proposition 1 (Revenue Equivalence). The expected revenue in any symmetric, incentive compatible mechanism where the participation constraint is binding for the lowest type (i.e. the lowest type obtains zero utility) is given by

$$
n \int_{\Theta} H(p(\theta), \theta) f(\theta) d \theta
$$

where the "virtual utility" $H:[0,1] \times[\underline{\theta}, \bar{\theta}] \rightarrow \mathbb{R}$ is defined by

$$
H(p, \theta):=h(p, \theta)-\frac{\partial h(p, \theta)}{\partial \theta} \times \frac{1-F(\theta)}{f(\theta)} .
$$

It is convenient to reformulate the problem in terms of the quantile $t=F(\theta)$ associated with a type $\theta .{ }^{14}$ For quantile $t$ we define the probability $q(t)=p\left(F^{-1}(t)\right)$ and the associated virtual utility

$$
G(q, t):=H\left(q, F^{-1}(t)\right)
$$

As $\frac{d \theta}{d t}=\frac{1}{f \circ F^{-1}(t)}$, integration by substitution of (4) yields the following equivalent characterization of expected revenue.

Expressed in terms of quantiles, the above revenue equivalence result yields:

Corollary 1. The expected revenue in any symmetric, incentive compatible mechanism where the participation constraint is binding for the lowest type is given by

$$
n \int_{0}^{1} G(q(t), t) d t
$$

\footnotetext{
${ }^{12}$ As the mechanism is symmetric, the definition of $p$ is independent of the agent's identity $i$.

${ }^{13}$ The result below also holds witout any symmetry assumptions.

${ }^{14}$ Since $F$ has a strictly positive density, $F^{-1}$ is well defined.
} 


\subsection{The Resource Constraint}

The non-linearity of the expected revenue $G$ in the allocation probability $q$ precludes a standard point-by-point maximization approach: since the revenue formula is not additively separable across realizations of types, changing the ex-post allocation at one type profile affects revenue from other type profiles. The crucial constraint becomes the feasibility (or resource) constraint: what expected interim allocations functions can be obtained from feasible, symmetric mechanisms? This question has been initially addressed (in the framework of a single object auction) by Matthews (1984), Maskin and Riley (1984), and Border (1991). ${ }^{15}$ The following, more recent result characterizes the set of feasible interim allocation rules for multi-unit auctions with one-dimensional types:

Proposition 2 (Che, Kim \& Mierendorff (2013), Corollary 4). Consider an auction with $n$ bidders where $m$ units are available. A symmetric, monotone interim allocation rule $q$ : $[0,1] \rightarrow[0,1]$ is a reduced form of a feasible allocation rule where no agent obtains more than $k$ objects if and only if, for each $t \in[0,1]$ it holds that

$$
n \int_{t}^{1} q(s) d s \leq \sum_{i=0}^{n} \min \{i \cdot k, m\}\left(\begin{array}{c}
n \\
i
\end{array}\right)(1-t)^{i} t^{n-i}
$$

We use a novel Lemma that connects the Che, Kim \& Mierendorff characterization to the concept of (weak) majorization: ${ }^{16}$

Lemma 2. It holds that

$$
\sum_{i=0}^{n} \min \{i, m\}\left(\begin{array}{c}
n \\
i
\end{array}\right) t^{n-i}(1-t)^{i}=n \int_{t}^{1} \phi_{m, n}(z) d z
$$

where $\phi_{m, n}(t)$ is the probability that at most $m-1$ out of $n-1$ agents have a type larger than the type associated with the quantile $t$ :

$$
\phi_{m, n}(t):=\sum_{i=0}^{m-1}\left(\begin{array}{c}
n-1 \\
i
\end{array}\right) t^{n-1-i}(1-t)^{i}
$$

The function $\phi_{m, n}$ is increasing in $t$ and $m$, and decreasing in $n$. Furthermore, $\int_{0}^{1} \phi_{m, n}(t) d t=$

\footnotetext{
${ }^{15}$ These papers were written without connections to the earlier mathematical literature on the existence of measures with given marginals, e.g. Strassen (1965).

${ }^{16}$ For a characterization of a single-object reduced form auction in terms of second-order stochastic dominance see Hart and Reny (2015).
} 
$\frac{m}{n}$.

Combining Proposition 2, Lemma 2 and Corollary 1, our revenue maximization problem is equivalent to the following problem:

Proposition 3 (Characterization of Revenue Maximizing Mechanisms). A symmetric mechanism is revenue maximizing if and only if the induced interim probability of receiving an object $q:[0,1] \rightarrow[0,1]$ solves

$$
\max _{q} n \int_{0}^{1} G(q(t), t) d t
$$

subject to:

$$
\begin{aligned}
& q \text { non-decreasing } \\
& \int_{t}^{1} q(z) d z \leq \int_{t}^{1} \phi_{m, n}(z) d z \quad \text { for all } t \in[0,1]
\end{aligned}
$$

where $t=F^{-1}(\theta), G$ is defined in (6) and where $\phi_{m, n}$ is defined in (9).

\subsection{Majorization and the Fan-Lorentz Inequality}

In order to deal with the resource constraint (12), we first recall several concepts and results from the theory of majorization. For non-decreasing $q, \bar{q} \in L^{1}(0,1)$ we say that $\bar{q}$ majorizes $q$, denoted by $q \prec \bar{q}$ if the following two conditions hold: ${ }^{17}$

$$
\begin{aligned}
\int_{t}^{1} q(v) d v & \leq \int_{t}^{1} \bar{q}(v) d v \text { for all } t \\
\int_{0}^{1} q(t) d t & =\int_{0}^{1} \bar{q}(t) d t .
\end{aligned}
$$

We say that $\bar{q}$ weakly majorizes $q$, denoted by $q \prec_{w} \bar{q}$ if the first condition above holds (but not necessarily the second). If $q \prec_{w} \bar{q}$ it is easily seen that there exists $q^{\prime} \leq \bar{q}$ such that $q \prec q^{\prime}$.

The set

$$
\Omega(\bar{q}):=\{q: q \prec \bar{q}\}
$$

called the orbit of $\bar{q}$, is weakly compact and convex. In particular, by Bauer's Maximum Principle (1958), a continuous, convex functional on $\Omega(\bar{q})$ attains its maximum on an extreme

\footnotetext{
${ }^{17}$ The definition can also be applied to possibly non-monotonic functions $q, \bar{q} \in L^{1}(0,1)$ if $q, \bar{q}$ in $(13)$ are replaced by their non-decreasing re-arrangements.
} 
point of $\Omega(\bar{q})$. Recall that an extreme point of a convex set $A$ is a point $x \in A$ that cannot be represented as a convex combination of two other points in $A^{18}$.

Fan and Lorentz (1954) identify a set of convex functionals such that all of them attain their maximum on $\Omega(\bar{q})$ at $\bar{q}$.

Proposition 4 (Fan-Lorentz Theorem). Let $L:[0,1] \times[0,1] \rightarrow \mathbb{R}$ be such that $L(q, t)$ is convex in $q$ and super-modular in $(q, t)$. Let $q, \bar{q}:[0,1] \rightarrow[0,1]$ be two non-decreasing functions such that $q \prec \bar{q}$. Then

$$
\int_{0}^{1} L(q(t), t) d t \leq \int_{0}^{1} L(\bar{q}(t), t) d t
$$

We next introduce a regularity assumption ensuring that the Fan-Lorentz conditions are satisfied by our expected revenue functional.

Definition 1 (Convex-Supermodularity). We say that the environment is "convex supermodular" (CSM) if the virtual utility $H(p, \theta)$ defined in (5) is convex in $p$ and super-modular in $(p, \theta)$.

\subsection{Illustrations}

Auction without Investments In the standard model without investments we have

$$
h\left(p_{i}, \theta_{i}\right)=p_{i} \theta_{i} \text { and } H(p, \theta)=p\left(\theta-\frac{1-F(\theta)}{f(\theta)}\right) .
$$

$H$ is linear and hence (weakly) convex in $p$. It is super-modular in $(p, \theta)$ if and only if the standard virtual value $J(\theta)=\theta-\frac{1-F(\theta)}{f(\theta)}$ is non-decreasing. Thus, in this case, our definition of a CSM environment reduces to the definition of a "regular" environment in Myerson (1981).

Investments with Additively Separable Values Assume that $v(a, \theta)=a+\theta$ where $a \in A \subseteq \mathbb{R}_{+} \cdot{ }^{19}$ Taking an arbitrary optimal selection for the action $a^{*}(p) \in \arg \max _{a \in A}[p(a+$ $\theta)-c(a)$ ] yields that

$$
h(p, \theta)=p \theta+p a^{*}(p)-c\left(a^{*}(p)\right)=p \theta+g(p),
$$

\footnotetext{
${ }^{18}$ Formally $x \in A$ is an extreme point of $A$ if $x=\alpha y+(1-\alpha) z$, for $z, y \in A$ and $\alpha \in[0,1]$ imply together that $y=x$ or $z=x$.

${ }^{19}$ While formally the set of actions is not compact here this is inconsequential: this assumption was only used to ensure that $h$ is well defined, which we verify here directly.
} 
where $g(p):=p a^{*}(p)-c\left(a^{*}(p)\right)$. As $h$ is convex and increasing in $p$ (see Lemma 1), the function $g$ is convex. Furthermore, $g(0)=0$ due to the existence of the costless action $a=0$. By plugging in (5) we obtain that

$$
H(p, \theta)=p J(\theta)+g(p)
$$

where $J(\theta)=\theta-\frac{1-F(\theta)}{f(\theta)}$ is the standard virtual value. As $g$ is convex, it follows immediately that $H$ is convex in $p$. Furthermore, if $J$ is non-decreasing then $H$ is super-modular, and thus the environment is CSM .

Investments with Multiplicative Values. Assume that $v(a, \theta)=a \theta$ where $a \in A=\mathbb{R}_{+}$ and that $c(a)=b \frac{a^{l}}{l}$ where $l>1$ and $b>0$. This yields that

$$
h(p, \theta)=\max _{a \in A}\left(p a \theta-b \frac{a^{l}}{l}\right)=\frac{l-1}{l}\left(\frac{p^{l} \theta^{l}}{b}\right)^{\frac{1}{l-1}} .
$$

and, by plugging in (5), that

$$
H(p, \theta)=p^{\frac{l}{l-1}}\left(\frac{\theta}{b}\right)^{\frac{1}{l-1}} K(\theta)
$$

where $K(\theta):=\frac{l-1}{l} \theta-\frac{1-F(\theta)}{f(\theta)}$. Assume now that $K$ is increasing (a sufficient condition is the standard monotone hazard rate condition). Assuming $\Theta=[0, \bar{\theta}]$, we obtain that $K(0)=-\frac{1}{f(0)}<0$ and that $H(p, \theta) \geq 0$ implies that $K(\theta) \geq 0$. Hence, $\max \{H(p, \theta), 0\}$ is convex in $p$ and super-modular in $(p, \theta)$. As in the standard case without investments, this variation from the case where $H(p, \theta)$ is itself convex and super-modular is inconsequential ${ }^{20}$, and the same methods/result apply.

Fixed Entry Costs This is also a multiplicative case, but there are only two actions: Enter $(a=1)$ and Stay Out $(a=0)^{21}$. We have $v\left(a_{i}, \theta_{i}\right)=a_{i} \theta_{i}$ and $c\left(a_{i}\right)=c a_{i}$ where

\footnotetext{
${ }^{20}$ If $J(\theta)=\theta-\frac{1-F(\theta)}{f(\theta)}$ decreases for some types to which $J$ assigns a negative virtual value, then, as those types are excluded, their virtual value plays no role in determining the optimal allocation.

${ }^{21}$ In contrast to our general model, the action $a$ is here observable, and the designer could condition the allocation/the transfers on it. Yet, this instrument is not useful since the allocation and transfers are only relevant if this bidder chooses action $a=1$. Thus, without loss. we can restrict attention to the mechanisms we already analyzed.
} 
$c>0 .{ }^{22}$ We obtain

$$
h(p, \theta)=\max \{p \theta-c, 0\} \text { and } \frac{\partial h(p, \theta)}{\partial \theta}=\left\{\begin{array}{l}
0 \text { if } p \theta<c \\
p \text { if } p \theta>c
\end{array} .\right.
$$

Thus

$$
H(p, \theta)=\left\{\begin{array}{ll}
0 & \text { if } p \theta<c \\
p\left(\theta-\frac{1-F(\theta)}{f(\theta)}\right)-c & \text { if } p \theta>c
\end{array} .\right.
$$

Assuming an increasing virtual value $J(\theta)=\theta-\frac{1-F(\theta)}{f(\theta)}$, note that $p J(\theta)-c \geq 0$ implies $p \theta \geq c$, and thus that $\max \{0, H(p, \theta)\}$ is convex in $p$ and super-modular in $(p, \theta)$.

\section{Optimal Mechanisms}

\subsection{The Optimal Symmetric Mechanism}

We now derive the optimal mechanism among symmetric ones using the Fan-Lorentz Theorem. To simplify notation, let

$$
\psi_{m, n}(\theta):=\phi_{m, n}(F(\theta))
$$

denote the interim probability with which an agent of type $\theta$ receives an object when $m$ objects are allocated efficiently among $n$ agents. We also denote by $a_{m, n}(\theta)$ the optimal action for type $\theta$ under allocation $\psi_{m . n}$ :

$$
a_{m, n}(\theta) \in \underset{a \in A}{\arg \max } \psi_{m, n}(\theta) v(a, \theta)-c(a)
$$

Theorem 1. Suppose the environment is convex super-modular. Then, the revenue maximizing scheme among all symmetric mechanisms allocates the $m$ objects to the agents with the highest types, conditional on these exceeding a threshold $\theta_{m, n}^{*} \equiv \theta^{*}$, where $\theta^{*}$ is the unique solution to $H\left(\psi_{m, n}\left(\theta^{*}\right), \theta^{*}\right)=0$, whenever such a type exists. ${ }^{23}$ In the optimal mechanism, the expected utility of the lowest type, $\underline{\theta}$ is zero.

The intuition for the proof of Theorem 1 (see Appendix) is as follows. First, in a CSM environment the quantile virtual value $G(q, t)$ is convex and super-modular in $(q, t)$ (Lemma

\footnotetext{
${ }^{22}$ Such auction environments are studied by Menezes and Monteiro (2000) and by Celik and Yilankaya (2009) - the latter authors also study asymmetric mechanisms.

${ }^{23}$ If $H\left(\psi_{m, n}(\theta), \theta\right)>0$ for any $\theta \in[\underline{\theta}, \bar{\theta}]$ we set $\theta^{*}=\underline{\theta}$, while if $H\left(\psi_{m, n}(\theta), \theta\right)<0$ for any $\theta \in[\underline{\theta}, \bar{\theta}]$ we set $\theta^{*}=\bar{\theta}$.
} 
4). Thus, the functional $q \mapsto \int_{0}^{1} G(q(t), t) d t$ is convex and it therefore attains a maximum on an extreme point of the set of monotone functions that satisfy the constraint (12). This constraint says that $q$ is weakly majorized by the allocation probability $\phi_{m, n}$. In turn, this implies that $q$ is majorized by a function of the form $t \mapsto 1_{\left\{t \geq t^{*}\right\}} \times \phi_{m, n}(t)$. Hence, by the Fan-Lorentz inequality, an interim probability of the form $t \mapsto 1_{\left\{t \geq t^{*}\right\}} \times \phi_{m, n}(t)$ maximizes revenue. The optimality of the cutoff type $\theta_{m, n}^{*} \equiv \theta^{*}$ follows because the expected revenue is quasi-concave in the cutoff $\theta^{*}$, and because $H\left(\psi_{m, n}\left(\theta^{*}\right), \theta^{*}\right)=0$ is the relevant first-order condition. This condition can be equivalently expressed as

$$
\psi_{m, n}\left(\theta^{*}\right) v\left(a_{m, n}\left(\theta^{*}\right), \theta^{*}\right)-c\left(a_{m, n}\left(\theta^{*}\right)\right)-\frac{1-F\left(\theta^{*}\right)}{f\left(\theta^{*}\right)} \psi_{m, n}\left(\theta^{*}\right)\left(\frac{\partial v\left(a_{m, n}\left(\theta^{*}\right), \theta^{*}\right)}{\partial \theta}\right)=0 .
$$

Here the probability of getting a unit affects the investment incentives, and hence indirectly influences the virtual value of every agent. The increase in virtual value (due to an increase in the probability of obtaining an unit) is more substantial for an agent with a higher type. Compared to the case where values are exogenous, this increases the advantage of allocating the units to agents with higher types, conditional on their virtual values being non-negative. It is thus not revenue maximizing to give the object to a lower type (i.e., withhold it from a higher type $)^{24}$.

\subsection{When is a Symmetric Mechanism (Not) Optimal?}

In this section, we identify a sufficient (and almost necessary) condition under which the optimal mechanism is symmetric, even if asymmetric mechanisms are allowed. ${ }^{25}$

Despite all bidders being ex-ante symmetric, and contrasting the case with exogenous valuations, the seller may find it profitable to employ an asymmetric mechanism ${ }^{26}$. To see this, note that, on the one hand, a discriminatory treatment decreases the payment that can be extracted from bidders with an exogenously given distribution of values. On the other hand, by arbitrarily choosing one bidder and giving him an advantage over competitors, the seller can further increase this particular bidder's investment incentives and shift his postinvestment value upwards. If the investment technology significantly affects values, it may

\footnotetext{
${ }^{24}$ We note that, outside the convex supermodular case, the quest for optimality involves other extreme points.

${ }^{25}$ For an interesting discussion of symmetric vs asymmetric auctions in a setting with exogenous values see Deb and Pai (2017).

${ }^{26}$ The general revenue maximizing mechanism maximizes the expected sum of virtual values under an asymmetric Border constraint (see also the proof of Theorem 2 in the Appendix)
} 
be worthwhile for the seller to sacrifice some ex-post rent in exchange for a higher ex-ante investment level.

The following Theorem identifies the exact condition under which the first effect dominates, rendering symmetric mechanisms overall optimal.

Theorem 2. Let $m=1$ and $n=2$ and suppose that the environment is convex supermodular. Let $p_{1}, p_{2}$ be the interim probabilities with which agent $i=1,2$ receive an object in a feasible, potentially asymmetric, mechanism. Suppose that $p_{1}$ and $p_{2}$ are piecewise continuously differentiable with a finite number of jumps, and that they induce an optimal mechanism. If for every $\theta \in[\underline{\theta}, \bar{\theta}]$

$$
\frac{\partial^{2} H(F(\theta), \theta)}{\partial p \partial \theta}>f(\theta) \frac{\partial^{2} H(F(\theta), \theta)}{\partial^{2} p}
$$

then the revenue maximizing mechanism is symmetric, i.e. $p_{1}=p_{2} \cdot{ }^{27}$

Roughly speaking, $\frac{\partial^{2} H}{\partial p \partial \theta}(F(\theta), \theta)$ measures the seller's marginal gain from allocating to a higher type, while $f(\theta) \frac{\partial^{2} H}{\partial^{2} p}(F(\theta), \theta)$ measures convexity - this relates to the seller's marginal incentive to replace a symmetric allocation by an asymmetric one. When the former term is uniformly larger, the seller wants to use the mechanism that is the most efficient, i.e. a symmetric auction.

Condition 15 is almost necessary: if the unique revenue maximizing mechanism always allocates the object to the agent with the higher type, provided that his type exceeds a threshold $\theta^{*}$, then it must be the case that $\frac{\partial^{2} H}{\partial p \partial \theta}(F(\theta), \theta)>f(\theta) \frac{\partial^{2} H}{\partial^{2} p}(F(\theta), \theta)$ on $\left(\theta^{*}, \bar{\theta}\right]$. When this condition fails, the optimal mechanism may be asymmetric.

The proof of Theorem 2 proceeds as follows: We first show that, given an interim probability with which agent 1 receives the object, the set of feasible interim probabilities for agent 2 is characterized by a majorization condition involving the allocation of agent 1 . This step is based on a generalization of Young's inequality. Next, we use the Fan-Lorentz Theorem to characterize the optimal allocation to agent 2 as a function of the allocation to agent 1 . This reduces the problem to the one of finding a solution to an optimization problem involving only the allocation of agent 1 . We solve this problem using the variational Euler-Lagrange principle.

\footnotetext{
${ }^{27}$ We consider piecewise continuously differentiable allocation probabilities with a finite number of jumps in order to use calculus of variations arguments. It is common to restrict attention to this class of mechanism when dealing with optimal control problem with jumps in the state variable (see, for example, Seierstad and Sydsæter (1987), p 196, Theorem 7).
} 
We next provide two simple examples that illustrate when condition 15 holds, and the structure of the optimal asymmetric (and hence overall) mechanism when it fails. ${ }^{28}$

Example 1. Let $v(a, \theta)=a \theta, c(a)=b \frac{a^{l}}{l}$ where $l>1, b>0$ and assume that $m=1$ and $n=2$. Recall that the virtual utility $H$ is given here by

$$
H(p, \theta)=H(p, \theta)=p^{\frac{l}{l-1}}\left(\frac{\theta}{b}\right)^{\frac{1}{l-1}} K(\theta)
$$

where $K(\theta)=\frac{l-1}{l} \theta-\frac{1-F(\theta)}{f(\theta)}$. Thus, condition 15 holds whenever $K(\theta)$ is increasing, and $F$ is concave.

Example 2. Let $v(a, \theta)=\theta+a$ and $c(a)=\frac{b}{2} a^{2}$, where $b>0$ and assume that $m=1$ and $n=2$. In this case, the virtual utility $H$ is given by

$$
H(p, \theta)=p(\theta) J(\theta)+\frac{1}{2 b} p^{2}(\theta) .
$$

Thus, condition 15 is equivalent to requiring that $J(\theta)-\frac{1}{b} F(\theta)=\theta-\frac{1-F(\theta)}{f(\theta)}-\frac{1}{b} F(\theta)$ is increasing. For example, when types are uniformly distributed on [0,1], condition 15 holds if and only if the investment cost is large enough, i.e. $b>\frac{1}{2}$.

In the above example, investment is more costly when $b$ is relatively large, and thus it plays a smaller role in determining agents' final value. In this case, the need to elicit private information dominates. When $b$ is small, agents have strong incentives to invest and their ex-ante private types contribute less to their final values. The need to increase agents' investment incentives becomes more important, and thus a discriminatory mechanism becomes desirable. If types are uniformly distributed on $[0,1]$ and if $b<\frac{1}{2}$, then $J(\theta)-\frac{1}{b} F(\theta)$ is decreasing on $[0,1]$, i.e., for every $\theta \in[0,1]$,

$$
\frac{\partial^{2} H(F(\theta), \theta)}{\partial p \partial \theta}<f(\theta) \frac{\partial^{2} H(F(\theta), \theta)}{\partial^{2} p} .
$$

In this case, the optimal mechanism is asymmetric. In the proof of Theorem 2, we show that an optimal allocation rule requires the seller to either : 1) keep the object; or 2) assign the object efficiently; or 3) assign the object to a randomly selected but pre-determined agent (referred to as the "favored" agent). Since the two bidders are ex-ante symmetric, it

\footnotetext{
${ }^{28}$ Early examples for the optimality of asymmetric mechanisms in settings with entry costs and additive valuations are in Celik and Yilankaia (2009) and Goeree (2015), respectively.
} 
is without loss of generality to always appoint agent 1 as the favored agent. If condition 15 holds, the efficient allocation rule performs better than random favoritism. When the opposite condition holds, similar arguments to those in the proof for Theorem 2 show that the optimal allocation may consist of the same three regions, but random favoritism performs better than efficient allocation. Hence, the optimal mechanism requires the seller to allocate the object to agent 1 as long as his type exceeds a threshold; otherwise, she allocates to agent 2 provided that his type exceeds a (possibly different) threshold. This asymmetric optimal mechanism can be implemented by a sequential posted-price scheme with favoritism: the seller first makes a take it-or-leave-it offer to agent 1. If she is turned down, she makes another take-it-or-leave-it offer to agent 2 (at a possibly different price). Finally, if neither condition 15 nor its converse hold, the optimal mechanism takes a dynamic, hybrid form that combines an auction with sequential posted-prices ${ }^{29}$.

\subsection{Restricting Entry}

Even if the symmetric optimal mechanism need not be the overall optimal one, the designer may be constrained by the legal system to use non-discriminatory schemes. By invoking various technical requirements, minimal standards or financial viability tests, etc., it is often possible to artificially limit the number of participants in the auction while still treating symmetrically those bidders that do participate (See Rogerson (1994) for concrete examples in defence procurement).

In a standard auction with exogenous valuations limiting participation is never beneficial. But, such a measure may be revenue-improving here: there is an additional positive effect, caused by the upwards adjustment of investments made by those bidders that are selected to participate.

Since we characterized the optimal symmetric mechanism for any number of bidders, we can easily determine the optimal number of bidders in a symmetric mechanism. This number necessarily depends on the particular parameters and functional forms of the values and costs.

In the multiplicative case with a single object, the expected revenue is monotonically increasing in the number of agents under relatively general assumptions (recall also Example 1 where we looked at the case with two agents):

\footnotetext{
${ }^{29}$ We refer interested readers to Zhang (2018) for formal proofs and more examples.
} 
Lemma 3. Assume that $m=1$. Assume further that $v(a, \theta)=a \theta, a \in A=\mathbb{R}_{+}, c(a)=b \frac{a^{l}}{l}$ where $l>1, b>0$, that $K(\theta)=\frac{l-1}{l} \theta-\frac{1-F(\theta)}{f(\theta)}$ is increasing, and that $F$ is concave. Then, the expected revenue increases in the number of agents $n$.

In contrast, for the additively separable investment case, restricting entry may be profitable when $b$ is small, i.e., when the investment cost is low. Then, an agent's ex-ante type plays a smaller role in determining her final value, and thus the need to encourage investments dominates the need to select an ex-ante more suitable agent. This observation is consistent with that in Example 2, i.e., an asymmetric mechanism becomes optimal when $b<\frac{1}{2}$.

Example 3. Let $m=1$, and consider the additively separable environment with $c(a)=b \frac{a^{2}}{2}$ where $b>0$. Then $H(p, \theta)=J(\theta) p+\frac{p^{2}}{2 b}$. Assuming uniformly distributed types $(\theta \sim U[0,1])$, the expected revenue is

$$
E R(n)=\frac{n-1}{n+1}-\frac{2 n}{n+1} \theta^{*^{n+1}}+\theta^{*^{n}}+\frac{n}{2 b} \frac{1-\theta^{*^{2 n-1}}}{2 n-1}
$$

where $\theta_{n}^{*} \equiv \theta^{*}$ solves

$$
2 \theta^{*}-1+\frac{\left(\theta^{*}\right)^{n-1}}{2 b}=0 .
$$

If the investment cost is low enough, the revenue sometimes decreases in the number of agents (c.f. Figure 2). But, the marginal expected revenue

$$
\frac{\partial E R(n)}{\partial n}=\frac{2\left(1-\theta^{*^{n+1}}\right)}{(n+1)^{2}}-\frac{\left(1-\theta^{*^{2 n-1}}\right)}{2 b(2 n-1)^{2}}-\frac{2 n \theta^{*^{n+1}}}{n+1} \ln \theta^{*}+\theta^{*^{n}} \ln \theta^{*}-\frac{n \theta^{*^{2 n-1}}}{(2 n-1) b} \ln \theta^{*}
$$

is always positive for sufficiently high $b .^{30}$ In that case, the cost of investing becomes prohibitive, and we approach the standard setting without investments where revenue is always increasing in the number of agents. To see this observe that $\lim _{b \rightarrow \infty} \theta_{n}^{*}=1 / 2$, and hence

$$
\lim _{b \rightarrow \infty} \frac{\partial E R(n)}{\partial n}=\frac{2\left(1-(0.5)^{n+1}\right)}{(n+1)^{2}}-\frac{2 n(0.5)^{n+1}}{n+1} \ln 0.5+(0.5)^{n} \ln 0.5>0 .
$$

The above example shows that the celebrated result due to Bulow and Klemperer (1996) need not hold in our environment. That result states that, in the setting without investments,

\footnotetext{
${ }^{30}$ We look here at the natural extension of $E R$ to the real line which allows us to express the derivative with respect to the number of agents.
} 


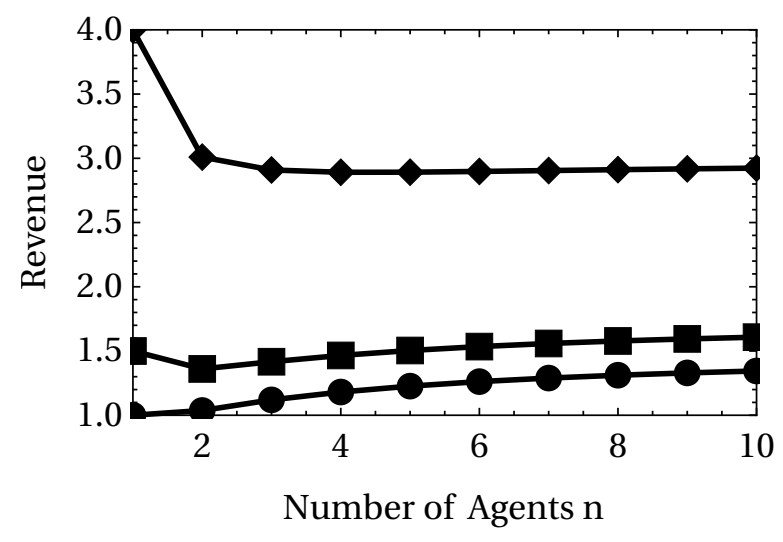

Figure 2: Revenue for the additively separable environments with quadratic costs $c(a)=b \frac{a^{2}}{2}$ and $\theta \sim U([0,1])$ in the optimal mechanism for $b=1 / 2$ (circles), $b=1 / 3$ (squares), $b=1 / 8$ (diamonds).

the seller is always better-off by attracting an additional bidder and setting a zero reserve price rather than setting the optimal reserve price when facing one less bidder. Here the seller may be worse-off if an additional bidder participates and if a symmetric mechanism is used. In particular, this implies that a symmetric mechanism is sub-optimal if the revenue is non-monotonic in the number of bidders

\section{Implementation via Standard Auctions}

In this Section we analyze two widely used, standard auction formats that implement the symmetric, revenue maximizing allocation in the model with costly investments. We assume throughout that the environment is convex and super-modular.

\subsection{The Uniform-Price Auction}

In the uniform $(m+1)$-price auction with a reserve price $\mathcal{R}$, all winners (there can be at most $m$ of them) pay the same price: the maximum between the $m+1$ highest bid and the reserve. Recall that $a_{m, n}$ is the optimal action in a $(m+1)$-price auction without reserve price

$$
a_{m, n}(\theta) \in \underset{a \in A}{\arg \max } \psi_{m, n}(\theta) v(a, \theta)-c(a) .
$$

The equilibrium strategy in the uniform-price auction with $n$ bidders, $m$ objects and reserve price $\mathcal{R}$ is straightforward: agents with type above a cut-off type $\theta_{m, n, \mathcal{R}}$ participate 
in the auction and make a non-zero bid. Every type of agent who participates chooses an optimal action $a_{m, n, \mathcal{R}}$ that is equal to the action $a_{m, n}(\theta)$ this type would have chosen in a $(m+1)$-price auction without reserve:

$$
a_{m, n, \mathcal{R}}(\theta)= \begin{cases}a_{m, n}(\theta) & \text { if } \theta \geq \theta_{m, n, \mathcal{R}} \\ 0 & \text { else }\end{cases}
$$

The critical type $\theta_{m, n, \mathcal{R}}$ - the lowest type who makes a non-zero bid - is indifferent between participating and not participating in the auction, i.e., his investment cost equals the expected gain from potentially winning an unit in the auction. This type solves:

$$
\psi_{m, n}\left(\theta_{m, n, \mathcal{R}}\right)\left[v\left(a_{m, n}\left(\theta_{m, n, \mathcal{R}}\right), \theta_{m, n, \mathcal{R}}\right)-\mathcal{R}\right]=c\left(a_{m, n}\left(\theta_{m, n, \mathcal{R}}\right)\right)
$$

The bid submitted by each participating type $\theta>\theta_{m, n, \mathcal{R}}$ is equal to the post-investment value from winning the auction, $v\left(a_{m, n}(\theta), \theta\right)$.

This profile of strategies (actions and bids) constitutes a standard Bayes-Nash equilibrium of the two-stage investment and bidding game. ${ }^{31}$ In contrast to the case without investment where the bid is independent of the number of goods or bidders, here the equilibrium bid responds both to demand (the number of bidders) and supply (the number of objects) via the investment decision $a_{m, n, \mathcal{R}}$ since the value $v\left(a_{m, n, \mathcal{R}}(\theta), \theta\right)$ increases in $\theta$ and in the number of objects $m$, and decreases in the number of bidders $n$.

Remark. There is a "jump" in the equilibrium bidding function at the reserve price: the type $\theta_{m, n, \mathcal{R}}$ given in (17) submits a bid that exceeds the reserve price $\mathcal{R}$, or put differently, there exists an interval of types $\left[\theta_{1}, \theta_{2}\right]$ such that their value when investing optimally exceeds the reserve price, $v\left(a_{m, n, \mathcal{R}}(\theta), \theta\right)>\mathcal{R}$, yet these types refrain from bidding! For a type with post-investment value just above the reserve price, it is not profitable to invest and participate at the auction: in the best scenario where his type is among the $m$ highest, he gets close to zero utility from winning, but loses the initial investment, leading to an overall loss.

Combining these insights with Theorem 1 yields:

Corollary 2. Let $\theta^{*} \equiv \theta_{m, n}^{*}$ be the optimal cutoff defined in Theorem 1 , and let $\mathcal{R}^{*} \equiv \mathcal{R}_{m, n, \theta^{*}}^{*}$ solve

$$
\mathcal{R}^{*}=\frac{1-F\left(\theta^{*}\right)}{f\left(\theta^{*}\right)}\left(\frac{\partial v\left(a_{m, n}\left(\theta^{*}\right), \theta^{*}\right)}{\partial \theta}\right) .
$$

\footnotetext{
${ }^{31}$ This profile is not in dominant strategies: conditional on other bids, an agent gets to know whether he wins or not, and may prefer to change his investment decision.
} 
Then the uniform-price auction with reserve price $\mathcal{R}^{*}$ is revenue maximizing among symmetric mechanism.

\subsection{The Pay-Your-Bid Auction}

In the discriminatory Pay-Your-Bid Auction with reserve price, the $m$ agents that submit the highest bids (conditional on these being above the reserve) get the objects and pay their bids. Other agents pay nothing. The discriminatory Pay-Your-Bid Auction with a properly chosen reserve price also implements the revenue maximizing allocation. We first derive the equilibrium bidding strategies for the more general auction setting where bidders have preferences that are convex (rather than linear) in the allocation ${ }^{32}$.

Proposition 5 (Equilibrium of the Discriminatory Auction). Assume that each bidder $i$ with type $\theta_{i}$ has preferences over allocations p represented by $h\left(p, \theta_{i}\right)$ where $h$ satisfies all properties of Lemma 1, and assume that the seller uses a Pay-Your-Bid Auction with reserve price $\mathcal{R}$. Then the bidding strategies

$$
\beta\left(\theta_{i}\right)= \begin{cases}\mathcal{R} \frac{\psi_{m, n}\left(\theta^{\prime}\right)}{\psi_{m, n}\left(\theta_{i}\right)}+\frac{1}{\psi_{m, n}\left(\theta_{i}\right)} \int_{\theta^{\prime}}^{\theta_{i}} \frac{\partial \psi_{m, n}(z)}{\partial \theta} \frac{\partial h\left(\psi_{m, n}(z), z\right)}{\partial p} d z & \theta_{i} \geq \theta^{\prime} \\ 0 & \theta_{i}<\theta^{\prime}\end{cases}
$$

where $\theta^{\prime}=\theta_{m, n, \mathcal{R}}^{\prime}$ solves

$$
\frac{h\left(\psi_{m, n}\left(\theta^{\prime}\right), \theta^{\prime}\right)}{\psi_{m, n}\left(\theta^{\prime}\right)}=\mathcal{R}
$$

constitute a symmetric, pure-strategy Bayes-Nash equilibrium in the Pay-Your-Bid Auction among $n$ bidders for $m$ units.

For the setting where $h$ is derived from investments recall that

$$
h\left(\psi_{m, n}\left(\theta_{i}\right), \theta_{i}\right):=\max _{a_{i} \in A} p_{i} v\left(\psi_{m, n}\left(\theta_{i}\right), \theta_{i}\right)-c\left(a_{i}\right)
$$

and hence that

$$
\frac{\partial}{\partial p_{i}} h\left(\psi_{m, n}\left(\theta_{i}\right), \theta_{i}\right)=v\left(a^{*}\left(\psi_{m, n}\left(\theta_{i}\right), \theta_{i}\right), \theta_{i}\right)
$$

where $a^{*}\left(\psi_{m, n}\left(\theta_{i}\right), \theta_{i}\right)$ is an optimal selection. The rest of the construction follows along the same lines as for the uniform-price auction. Combining the above insights with Theorem 1

\footnotetext{
${ }^{32}$ This derivation is of independent interest since it covers cases where bidders do not have expected utility preferences. An alternative derivation can be obtained by a payoff equivalence argument.
} 
yields:

Corollary 3. Let $\theta^{*} \equiv \theta_{m, n}^{*}$ be the optimal cutoff defined in Theorem 1 , and let $\mathcal{R}^{*} \equiv \mathcal{R}_{m, n, \theta^{*}}^{*}$ solve

$$
\mathcal{R}^{*}=\frac{1-F\left(\theta^{*}\right)}{f\left(\theta^{*}\right)}\left(\frac{\partial v\left(a_{m, n}\left(\theta^{*}\right), \theta^{*}\right)}{\partial \theta}\right) .
$$

Then the pay-your-bid auction with reserve price $\mathcal{R}^{*}$ is revenue maximizing among symmetric mechanism.

\subsection{Comparative Statics}

In sharp contrast to the standard setting without investments, the optimal cutoff type and the optimal reserve price are influenced both by the number of agents (demand) and objects (supply), and move in opposite directions. From the definition of $\psi_{m, n}(\theta)=\phi_{m, n}(F(\theta))$, we know that $\psi_{m, n}(\theta)$ is increasing in $m$ and in $\theta$ and decreasing in $n$ - these three basic properties account for:

Proposition 6 (Comparative Statics for the Cutoff Type). Assume that the environment is convex super-modular (CSM). Then the optimal cutoff type $\theta_{m, n}^{*} \equiv \theta^{*}$ increases in the number of agents $n$ and decreases in the number of objects $m$.

An increase in the number of agents decreases the individual chance of each agent to receive an object. This, in turn, reduces the individual incentives to invest, the resulting values, and the revenue that can be obtained from each type. In particular, the type with zero virtual utility - that determines the optimal cutoff type $\theta^{*}$ - must be larger than the analogous type when there are less bidders. ${ }^{33}$ As they never receive an object, agents with types below $\theta^{*}$ choose the action with zero cost. The action chosen by higher types $\theta>\theta^{*}$ can be determined by maximizing their interim utility (given the interim probability with which they receive an object).

The above result and intuition do not carry over to the optimal reserve price (which is the same for both the Uniform-Price and the Pay-Your-Bid auctions). To see this, recall that

$$
\mathcal{R}_{m, n, \theta^{*}}^{*} \equiv \mathcal{R}^{*}=\frac{1-F\left(\theta_{m, n}^{*}\right)}{f\left(\theta_{m, n}^{*}\right)}\left(\frac{\partial v\left(a_{m, n}\left(\theta_{m, n}^{*}\right), \theta_{m, n}^{*}\right)}{\partial \theta}\right)
$$

The optimal reserve price renders the optimal cut-off type indifferent between participating and not participating: an increase in the number of agents increases the cutoff type, but, on

\footnotetext{
${ }^{33} \mathrm{An}$ inverse effect is at work when the number of objects increases.
} 
the other hand, this type must be compensated for the increase in competition by a reduction of the reserve price. Inverse effects are at work when the number of objects $m$ is variable.

Proposition 7 (Comparative Statics of the Optimal Reserve Price). Assume that the environment is convex super-modular (CSM). Assume further that $v$ is concave in $\theta$, and that $F$ has an increasing hazard rate with a differentiable density function. If $a_{m, n}\left(\theta_{m, n}^{*}\right)$ is decreasing in $n$ (increasing in $m$ ), then the optimal reserve price $\mathcal{R}_{m, n, \theta^{*}}^{*} \equiv \mathcal{R}^{*}$ decreases in the number of agents $n$ (increases in the number of objects $m$ ). ${ }^{34}$ Moreover, if $v(a, \theta)=d(a)+e(\theta)$, then the optimal reserve price $R_{m, n, \theta^{*}}^{*} \equiv R^{*}$ decreases in the number of agents $n$ (increases in the number of objects $m){ }^{35}$

This result should be compared to the standard "knife-edge" result obtained in the linear case, where the reserve price is non-responsive to changes in the number of bidders and objects: since in that case the optimal cutoff type and the optimal reserve price coincide, this non-response is indeed suggested by our results that display comparative statics in opposite directions for the more general model where these two objects do not coincide.

\subsubsection{Illustrations}

Additively separable investments Both the Uniform-Price and the Pay-Your-Bid auction with reserve price

$$
\mathcal{R}_{m, n}^{*}=\frac{1-F\left(\theta_{m, n}^{*}\right)}{f\left(\theta_{m, n}^{*}\right)}
$$

where $\theta_{m, n}^{*}$ satisfies

$$
\theta_{m, n}^{*}+\frac{g\left(\psi_{m, n}\left(\theta_{m, n}^{*}\right)\right)}{\psi_{m, n}\left(\theta_{m, n}^{*}\right)}=\frac{1-F\left(\theta_{m, n}^{*}\right)}{f\left(\theta_{m, n}^{*}\right)}
$$

maximize revenue among symmetric mechanisms. If the hazard rate $\frac{f(\theta)}{1-F(\theta)}$ is increasing, then the optimal reserve price increases in the number of objects and decreases in the number of bidders.

\footnotetext{
${ }^{34}$ The condition can be easily checked as it is stated in terms of the model's primitives, and of the solution to the individual investment problem

$$
a_{m, n}\left(\theta_{m, n}^{*}\right)=\arg \max _{a} \psi_{m, n}\left(\theta_{m, n}^{*}\right) v\left(a, \theta_{m, n}^{*}\right)-c(a)
$$

${ }^{35}$ For particular functional forms (see the multiplicative case below) the comparative static result holds even though the above assumptions are not satisfied.
} 
Multiplicative separable investments and monomial cost Assume that the function $K(\theta)=\frac{l-1}{l} \theta-\frac{1-F(\theta)}{f(\theta)}$ is increasing. Then both the Uniform-Price and the Pay-Your-Bid auctions with reserve price

$$
\mathcal{R}_{m, n}^{*}=\frac{l-1}{l}\left(\frac{\psi_{m, n}\left(\theta^{*}\right)\left(\theta^{*}\right)^{l}}{b}\right)^{\frac{1}{l-1}}
$$

where $\theta_{m, n}^{*}=\theta^{*}$ solves $K\left(\theta^{*}\right)=0$ maximize revenue among symmetric mechanisms. Since the critical cutoff $\theta^{*}$ is here independent of both $n$ and $m$, the optimal reserve price is decreasing in the number of bidders $n$ and increasing in the number of objects $m$.

For example, let $n=10, v(a, \theta)=a \theta, c(a)=\frac{a^{2}}{2}$, and assume that initial types are uniformly distributed on $[0,1]$. Then $H(p, \theta)=p^{2} \theta\left(\frac{3}{2} \theta-1\right)$. The revenue maximizing cutoff type is $\theta^{*}=\frac{2}{3}$ independently of $m, n$. However, when the number of objects on sale increases from 1 to 9 , the optimal reserve price (that induces the cutoff $\theta^{*}=\frac{2}{3}$ ) increases from $R_{1,10}^{*} \simeq 0.006$ to $R_{9,10}^{*} \simeq 0.222$. Using $R_{9,10}^{*}$ in a standard auction when $m=9$ yields an expected revenue of

$$
10 \int_{0.667}^{1}\left[1-(1-\theta)^{9}\right]^{2} \theta\left(\frac{3}{2} \theta-1\right) d \theta \simeq 0.741
$$

If the seller does not adjust the reserve price and continues to use $R_{1,10}^{*} \simeq 0.006<R_{9,10}^{*} \simeq$ 0.222 , then the (non-optimal) induced cutoff is $\theta_{9,10, R_{1,10}^{*}}=0.128$, and the expected revenue is only

$$
10 \int_{0.128}^{1}\left[1-(1-\theta)^{9}\right]^{2} \theta\left(\frac{3}{2} \theta-1\right) d \theta \simeq 0.135,
$$

an $80 \%$ decrease!

Fixed entry costs Assume that the standard virtual value $J(\theta)=\theta-\frac{1-F(\theta)}{f(\theta)}$ is increasing. Then both the Uniform-Price and the Pay-Your-Bid Auctions with reserve price

$$
\mathcal{R}_{m, n}^{*}=\frac{1-F\left(\theta_{m, n}^{*}\right)}{f\left(\theta_{m, n}^{*}\right)}
$$

where $\theta_{m, n}^{*}$ solves

$$
\psi_{m, n}\left(\theta_{m, n}^{*}\right)\left(\theta_{m, n}^{*}-\frac{1-F\left(\theta_{m, n}^{*}\right)}{f\left(\theta_{m, n}^{*}\right)}\right)=c
$$


maximize revenue among symmetric mechanisms. If the hazard rate $\frac{f(\theta)}{1-F(\theta)}$ is increasing, then the optimal reserve price is decreasing in the number of agents $n$ and increasing in the number of objects $m$.

\section{Welfare Maximization}

For comparison, we briefly discuss here the welfare maximizing mechanism, and first restrict attention to symmetric mechanisms.

Symmetric Mechanisms The above developed tools allow us to characterize the welfare maximizing mechanism in this class. Observe that the social welfare in a symmetric mechanism with allocation rule $p$ is

$$
n \int_{\Theta} h(p(\theta), \theta) f(\theta) d \theta
$$

and hence the conditions of Lemma 1 allow us to use Fan-Lorentz insight without any additional assumptions (since $h$ always satisfies CSM). Hence, the welfare maximizing, symmetric, direct mechanism allocates objects to the $m$ agents with the highest values. A simple indirect implementation of this allocation is an $(m+1)$-price auction without reserve price (see Section 5).

Proposition 8. The welfare maximizing symmetric allocation is implemented by an investment equilibrium in which agents use the welfare maximizing, symmetric investment strategies, followed by an $(m+1)$ price auction where the objects are allocated to the highest bidders.

Since an individual investment depends on the probability to get an object, we can compare the investments in the symmetric revenue maximizing mechanism to those in the symmetric welfare maximizing one: all types above the revenue maximizing cutoff type $\theta_{m, n}^{*}$ make the same investments in welfare- and revenue-maximizing mechanisms, while types below $\theta_{m, n}^{*}$ do not invest in the revenue maximizing mechanism, but make positive investment in the welfare maximizing one.

General Mechanisms The characterization of the welfare maximizing (potentially) asymmetric allocation rule is again complex. By using essentially the same argument as for Theorem 2, we obtain that the symmetric welfare maximizing mechanism for the case with 2 
bidders and 1 object is also the overall optimal one if, for all $\theta \in[\underline{\theta}, \bar{\theta}]$,

$$
\frac{\partial^{2} h(F(\theta), \theta)}{\partial p \partial \theta}>f(\theta) \frac{\partial^{2} h(F(\theta), \theta)}{\partial^{2} p} .
$$

If the opposite inequality holds for all $\theta \in[\underline{\theta}, \bar{\theta}]$, then a sequential posted-price scheme with favoritism is optimal. Note that a welfare maximizing seller will always allocate the object in a an optimal sequential posted-price scheme, while a revenue maximizing one may sometimes keep it.

Despite the difficulty of explicitly characterizing the optimal direct mechanism, it is now relatively easy to identify an indirect mechanism that implements the optimal allocation. Consider the Vickrey-Clarke-Groves (VCG) mechanism where each agent pays the expected externality he imposes on the other agents. In our context, this mechanism corresponds to a uniform $(m+1)$ price auction. Clearly, the $(m+1)$-price auction, which allocates to the agents with the $m$ highest ex-post valuations, is welfare-maximizing for every fixed vector of (potentially asymmetric) investments strategies. To show that it is an overall welfare maximizing mechanism, we need to show that it also induces the efficient investments as an equilibrium (see Appendix). This will follow from the insight that an agent gains by investing exactly when the investment increases the total welfare ${ }^{36}$.

Proposition 9. The welfare maximizing allocation is implemented by a (potentially asymmetric) investment equilibrium in which agents use the welfare maximizing investment strategies, followed by an $(m+1)$ price auction where the objects are allocated to the highest bidders.

It is easier to find an implementation for the welfare revenue mechanism than for a revenue maximizing mechanism because the VCG mechanism aligns the incentives of all agents with those of the designer, while no mechanism can achieve that for revenue maximization ${ }^{37}$.

\footnotetext{
${ }^{36}$ The logic underlying Proposition 9 is similar to the one in Rogerson (1992), Milgrom (2017) (Propositions 3.5 and 3.6) and Hatfield et. al. (2018) who establish similar results. Here each agent has to take an investment decision without knowing the other agents types while in Hatfield et. al. agents take decision contingent on other agents' types. Milgrom focuses on binary investment strategies.

${ }^{37}$ It is for exactly this reason that we know that the dynamic VCG mechanism maximizes welfare in very general dynamic settings (with potentially multidimensional and stochastically evolving types, see Bergemann and Välimäki (2010)) while a general characterization of the revenue maximizing mechanisms remains an open question.
} 


\section{Conclusion}

We have analyzed revenue maximization in a multi-unit auction framework where agents make, prior to the auction, costly investments that affect their values. The effective agents' utility functions become convex in probability of the physical allocation. Our main results employed a novel maximization approach, focused on a majorization inequality. We first identified the revenue maximizing allocation within the class of symmetric mechanisms, and illustrated how it can be implemented via standard auction formats. We then gave a condition ensuring that the optimal mechanism is symmetric in the two agent case (even if asymmetric mechanisms are allowed), and also illustrated the main features of optimal asymmetric mechanisms. Finally, we displayed novel comparative statics about the optimal reserve price.

\section{References}

[2004] Arozamena, L., \& Cantillon, E. (2004). Investment Incentives in Procurement Auctions. The Review of Economic Studies, 71(1), 1-18.

[1997] Bag, P. K. (1997). Optimal Auction Design and R\&D. European Economic Review, 41(9), 1655-1674.

[1958] Bauer, H. (1958): "Minimalstellen von Funktionen und Extremalpunkte I" Archiv der Mathematik 9, 389-393.

[2010] Bergemann, D. and Välimäki, J. (2010): "The dynamic pivot mechanism" Econometrica 78.2, 771-789.

[1991] Border, K. (1991): "Implementation of Reduced Form Auctions: A Geometric Approach," Econometrica 59(4), 1175-1187.

[1996] Bulow, J. and Klemperer, P. (1996): "Auctions vs Negotiations," The American Economic Review 86(1), 180-194.

[2009] Celik, G and Yilankaya, O. (2009): "Optimal Auctions with Simultaneous and Costly Participation", B.E. Journal of Theoretical Economics (Advances) 9(1), Article 24.

[2003] Che, Y. K., \& Gale, I. (2003). Optimal Design of Research Contests. American Economic Review, 93(3), 646-671. 
[2013] Che, Y.K., Kim, J. and Mierendorff, K. (2013): "Generalized Reduced Form Auctions: A Network-Flow Approach," Econometrica 81(6), 2487-2520.

[1990] Crawford, V. (1990): "Equilibrium without Independence," Journal of Economic Theory 50, 127-154.

[2017] Deb, R. and Pai, M. (2017): "Discrimination via symmetric auctions", American Economic Journal: Microeconomics 9(1), 275-314.

[2014] Directive 2014/24/EU of the European Parliament and of the Council of 26 February 2014 on public procurement and repealing Directive 2004/18/EC

[1954] Fan, K. and Lorentz, G.G. (1954): "An Integral Inequality," The American Mathematical Monthly 61(9), 626-631

[1991] Fudenberg, D. and Tirole, J. (1991): Game Theory, Cambridge: MIT Press.

[2009] Fullerton, R. L., \& McAfee, R. P. (1999). Auctioning Entry Into Tournaments. Journal of Political Economy, 107(3), 573-605.

[2013] Gershkov, A., Goeree J., Kushnir, A., Moldovanu, B. and Shi, X. (2013): "On the Equivalence of Bayesian and Dominant Strategy Implementation," Econometrica 81(1), 197-220.

[2015] Goeree, J. K. (2015): Lectures on mechanism design (with applications), unpublished lecture notes.

[1984] Guesnerie, R. and Laffont J.-J. (1984): "A Complete Solution to a Class of PrincipalAgent Problems with an Application to the Control of a Self-Managed Firm," Journal of Public Economics 25, 329-369.

[1929] Hardy, G. H., Littlewood J. E., and Polya G. (1929): "Some Simple Inequalities Satisfied by Convex Functions," Messenger Math 58, 145-152.

[2015] Hart, S. and Reny, P (2015): "Implementation of Reduced Form Mechanisms: a Simple Approach and a New Characterization," Economic Theory Bulletin 3(1), 1-8.

[2018] Hatfield, J., Kojima, F and Kominers, S. (2018): "Strategy-Proofness, Investment Efficiency, and Marginal Returns: An Equivalence," working paper. 
[2000] Jeitschko, T. Wolfstetter, E. (2000). "Auctions when bidders prepare by investing in ideas." Economics Letters 68(1), 61-66.

[1979] Kahneman, D. and Tversky, A. (1979): "Prospect Theory: An Analysis of Decision Under Risk," Econometrica 47(2), 263-291.

[1989] Karni, E. and Safra, Z. (1986): "Dynamic Consistency, Revelations in Auctions and the Structure of Preferences," Review of Economic Studies 56,421-434.

[1992] King, I., Welling, L., \& McAfee, R. P. (1992). Investment Decisions Under First and Second Price Auctions. Economics Letters, 39(3), 289-293.

[1979] Kreps, D. and Porteus, E. (1979): “Temporal von Neumann-Morgenstern and Induced Preferences," Journal of Economic Theory 20, 81-109.

[1984] Machina, M. (1984): "Temporal Risk and the Nature of Induced Preferences," Journal of Economic Theory 33, 199-231.

[2010] Manelli, A. and Vincent, D. (2010): "Bayesian and Dominant Strategy Implementation in the Independent, Private Values Model," Econometrica 78(6), 1905-1939.

[1984] Maskin, E. S. and Riley, J. (1984), "Optimal Auctions with Risk-Averse Buyers," Econometrica 52, 1473-1518.

[1984] Matthews, S. A. (1984), "On the Implementability of Reduced Form Auctions," Econometrica 52, 1519-1522.

[2000] Menezes, F. and Monteiro, P. (2000): "Auctions with Endogenous participation," Review of Economic Design 5, 71-89.

[2016] Mierendorff, K. (2016): "Optimal dynamic mechanism design with deadlines," Journal of Economic Theory 161, 190-222.

[2017] Milgrom, P. (2017) Discovering Prices, New York: Columbia University Press

[2002] Milgrom, P., \& Segal, I. (2002). Envelope Theorems for Arbitrary Choice Sets. Econometrica 70(2), 583-601.

[1994] Milgrom, P. and Shannon C. (1994) "Monotone Comparative Statics," Econometrica, $157-180$. 
[2011] Mitroi, F.C. and Niculescu, C.P. (2011): "An Extension of Young's Inequality," $A b$ stract and Applied Analysis, 1-18.

[1981] Myerson, R.B. (1981) "Optimal Auction Design," Mathematics of Operations Research, 6(1), 58-73.

[1984] Neilson, W. (1994) "Second Price Auctions without Expected Utility," Journal of Economic Theory 62, 136-151

[1996] Piccione, M., \& Tan, G. (1996). Cost-reducing Investment, Optimal Procurement and Implementation by Auctions. International Economic Review, 663-685.

[1982] Quiggin, J. (1982), "A Theory of Anticipated Utility," Journal of Economic Behavior \& Organization, 3(4), 323-343.

[1981] Riley, J. and Samuelson, W. (1981), "Optimal Auctions," American Economic Review, 71(3), 381-392.

[1992] Rogerson, W. P., (1992), "Contractual Solutions to the Hold-Up Problem," Review of Economic Studies, 59, issue 4, p. 777-793.

[1994] Rogerson, W. P. (1994)"Economic Incentives and the Defense Procurement Process." Journal of Economic Perspectives, 8 (4): 65-90.

[1992] Sagan, H. (1992): Introduction to the Calculus of Variations, New York: McGrawHill.

[1987] Seierstad, A. and Sydsæter, K. (1987): Optimal Control Theory with Economic Applications, North Holland.

[1965] Strassen, V. (1965): "The Existence of Probability Measures with Given Marginals," Mathematical Statistics 36, 423-439.

[2011] Vohra, R.V. (2011): Mechanism Design: A Linear Programming Approach, Cambridge: Cambridge University Press

[1992] Tan, G. (1992). Entry and R \& D in Procurement Contracting. Journal of Economic Theory, 58(1), 41-60.

[2011] Toikka, J. (2011) : "Ironing Without Control," Journal of Economic Theory, 146(6), $2510-2526$. 
[2007] Ye, L. (2007). "Indicative Bidding and a Theory of Two-Stage Auctions". Games and Economic Behavior 58(1), 181-207.

[2018] Zhang, M. (2018): "Auctions vs. Negotiations: Optimal Selling Mechanism with Endogenous Bidder Values," discussion paper, University of Bonn.

\section{Appendix: Proofs}

Proof of Lemma 1: $h$ is convex in $p$ as it is the maximum over functions that are linear in $p$. It is increasing in $p$ and $\theta$ as $p v(a, \theta)-c(a)$ is increasing in $p$ and $\theta$. By assumption, there exists an action 0 with $c(0)=0$ and the cost is always non-negative. Hence, $h(0, \theta)=$ $\max _{a \in A_{i}}-c(a)=0$. Finally, let $a^{*}\left(p_{i}, \theta_{i}\right)$ be an optimal selection for the problem given in (3). As $p_{i} v\left(a_{i}, \theta_{i}\right)-c\left(a_{i}\right)$ is super-modular in $\left(a_{i}, \theta_{i}\right)$ we can always pick an optimal selection $a^{*}\left(p_{i}, \theta_{i}\right)$ which is non-decreasing in $\theta_{i}$ (Theorem 4 in Milgrom and Shannon 1994). By the Envelope Theorem (see Milgrom and Segal (2002) Theorem 2), we obtain that $h$ is absolutely continuous in $p$ with (weak) derivative

$$
\frac{\partial}{\partial p_{i}} h\left(p_{i}, \theta_{i}\right)=v\left(a^{*}\left(p_{i}, \theta_{i}\right), \theta_{i}\right)
$$

As $a^{*}\left(p_{i}, \theta_{i}\right)$ is non-decreasing in $\theta_{i}$ and $v$ is non-decreasing in $a_{i}$ and $\theta_{i}$, the marginal utility associated with an increase in the probability of receiving the object, $\frac{\partial}{\partial p_{i}} h$, is non-decreasing in $\theta_{i}$. Thus, $h$ is super-modular in $\left(p_{i}, \theta_{i}\right)$.

Proof of Proposition 1: Denote by $p\left(\theta_{i}\right)$ the interim probability with which agent $i$ receives an object when she is of type $\theta_{i}$ and by

$$
u\left(\theta_{i}, \tilde{\theta}_{i}\right)=h\left(p\left(\tilde{\theta}_{i}\right), \theta_{i}\right)-\mathbb{E}\left[y\left(\tilde{\theta}_{i}, \theta_{-i}\right)\right]
$$

the interim utility of agent $i$ if she is of type $\theta_{i}$ but she misrepresents her type as $\tilde{\theta}_{i}$. Recall that we only consider here symmetric mechanisms, and thus the functions $p$ and $u$ are independent of the agents' identities- we drop the agent's sub-index $i$ for the remainder of the proof. Let $U(\theta)$ be the indirect utility of an agent of type $\theta$ who reports optimally

$$
U(\theta)=\max _{\tilde{\theta}} u(\theta, \tilde{\theta})
$$


Incentive compatibility and the envelope theorem yields that

$$
\frac{\partial U}{\partial \theta}=\frac{\partial u}{\partial \theta}=\frac{\partial h}{\partial \theta}
$$

The almost everywhere existence of $\frac{\partial h}{\partial \theta}$ and the following envelope formula follow by Milgrom and Segal (2002):

$$
U(\theta)=U(\underline{\theta})+\int_{\underline{\theta}}^{\theta} \frac{\partial h(p(z), z)}{\partial \theta} d z=\int_{\underline{\theta}}^{\theta} \frac{\partial h(p(z), z)}{\partial \theta} d z .
$$

The last equality follows from the binding participation constraint. The revenue from each type is then given by

$$
h(p(\theta), \theta)-U(\theta)=h(p(\theta), \theta)-\int_{\underline{\theta}}^{\theta} \frac{\partial h(p(z), z)}{\partial \theta} d z
$$

and thus the objective becomes

$$
\max _{p} \int_{\underline{\theta}}^{\bar{\theta}}\left(h(p(\theta), \theta)-\int_{\underline{\theta}}^{\theta} \frac{\partial h(p(z), z)}{\partial \theta} d z\right) f(\theta) d \theta .
$$

Using integration by parts, we obtain:

$$
\int_{\underline{\theta}}^{\bar{\theta}}\left(\int_{\underline{\theta}}^{\theta} \frac{\partial h(p(z), z)}{\partial \theta} d z\right) f(\theta) d \theta=\int_{\underline{\theta}}^{\bar{\theta}} \frac{\partial h(p(\theta), \theta)}{\partial \theta}[1-F(\theta)] d \theta
$$

so that the expected revenue from any agent is only a function of the interim probability $p$ with which an agent receives an object

$$
\int_{\underline{\theta}}^{\bar{\theta}}\left(h(p(\theta), \theta)-\frac{\partial h(p(\theta), \theta)}{\partial \theta} \times \frac{1-F(\theta)}{f(\theta)}\right) f(\theta) d \theta .
$$

Multiplying by the number of agents $n$ yields the result.

Proof of Lemma 2: The result follows from considering the right-hand-side of the Border 
constraint derived in Corollary 4 in Che, Kim \& Mierendorff (2013)

$$
\begin{aligned}
\frac{\partial}{\partial t} & \left(\sum_{i=0}^{n} \min \{i, m\}\left(\begin{array}{c}
n \\
i
\end{array}\right) t^{n-i}(1-t)^{i}\right)=\frac{\partial}{\partial t}\left(m-\sum_{i=0}^{m-1}(m-i)\left(\begin{array}{c}
n \\
i
\end{array}\right) t^{n-i}(1-t)^{i}\right) \\
& =-\sum_{i=0}^{m-1}(m-i)\left(\begin{array}{c}
n \\
i
\end{array}\right)\left[(n-i) t^{n-i-1}(1-t)^{i}-i t^{n-i}(1-t)^{i-1}\right] \\
& =\sum_{i=0}^{m-1}\left(\left[(m-i)\left(\begin{array}{c}
n \\
i
\end{array}\right) i t^{n-i}(1-t)^{i-1}\right]-\left[(m-i)\left(\begin{array}{c}
n \\
i
\end{array}\right)(n-i) t^{n-i-1}(1-t)^{i}\right]\right) \\
& =\sum_{i=0}^{m-1}\left(\left[(m-i-1)\left(\begin{array}{c}
n \\
i+1
\end{array}\right)(i+1) t^{n-i-1}(1-t)^{i}\right]-\left[(m-i)\left(\begin{array}{c}
n \\
i
\end{array}\right)(n-i) t^{n-i-1}(1-t)^{i}\right]\right) \\
& =\sum_{i=0}^{m-1} t^{n-i-1}(1-t)^{i}\left[(m-i-1)\left(\begin{array}{c}
n \\
i+1
\end{array}\right)(i+1)-(m-i)\left(\begin{array}{c}
n \\
i
\end{array}\right)(n-i)\right] \\
& =\sum_{i=0}^{m-1} t^{n-i-1}(1-t)^{i}\left[(m-i-1)\left(\begin{array}{c}
n \\
i
\end{array}\right)(n-i)-(m-i)\left(\begin{array}{c}
n \\
i
\end{array}\right)(n-i)\right] \\
& =-\sum_{i=0}^{m-1} t^{n-i-1}(1-t)^{i}\left(\begin{array}{c}
n \\
i
\end{array}\right)[n-i]=-n \sum_{i=0}^{m-1} t^{n-i-1}(1-t)^{i}\left(\begin{array}{c}
n-1 \\
i
\end{array}\right)=-n \phi_{m, n}(t) .
\end{aligned}
$$

Finally, we obtain that $\int_{0}^{1} \phi_{m, n}(t) d t$ is given by

$$
n \int_{0}^{1} \phi_{m, n}(t) d t=\left.\sum_{i=0}^{n} \min \{i, m\}\left(\begin{array}{c}
n \\
i
\end{array}\right) t^{n-i}(1-t)^{i}\right|_{t=0}=m
$$

Lemma 4. $G(0, t)=0$ for all $t \in[0,1]$. Furthermore, if the environment is CSM, then $G(q, t)$, expressed as a function of the quantile, is convex in $q$ and super-modular in $(q, t)$.

Proof of Lemma 4: Note first that

$$
G(0, t)=H\left(0, F^{-1}(t)\right)=h\left(0, F^{-1}(t)\right)-\frac{\partial h\left(0, F^{-1}(t)\right)}{\partial \theta} \times \frac{1-t}{f\left(F^{-1}(t)\right)} .
$$

As $h(0, \theta)=0$ by assumption, it follows that $\frac{\partial h\left(0, F^{-1}(t)\right)}{\partial \theta}=0$ and thus that $G(0, t)=0$. In a CSM environment $G(q, t)=H\left(q, F^{-1}(t)\right)$ is convex in $q$ since $H(p, \theta)$ is convex in $p$. Similarly, because $H$ is super-modular, because $F^{-1}$ is strictly increasing and because super-modularity is preserved under strictly monotone transformations, we obtain that $G$ is super-modular. 
Proof of Theorem 1: Since the number of agents and objects is fixed throughout the proof and thus there is no risk of confusion, we will drop the subindices, and write $\phi$ for $\phi_{m, n}$ throughout. Let $q^{*}(t)$ be the interim probability with which an agent of type $\theta=F^{-1}(t)$ receives an object in an optimal symmetric mechanism. Since in a symmetric mechanism no agent can receive the object with an ex-ante probability greater than $m / n$, we obtain that

$$
\int_{0}^{1} q^{*}(t) d t \leq \frac{m}{n}=\int_{0}^{1} \phi(t) d t
$$

Consequently, there exists a quantile $t^{*} \in[0,1]$ such that the ex-ante probability with which an agent receives an object in the optimal mechanism equals the probability with which an agent would have a type higher than $F^{-1}\left(t^{*}\right)$ and receive an object under the interim allocation probability $\phi$ :

$$
\int_{0}^{1} q^{*}(t) d t=\int_{t^{*}}^{1} \phi(t) d t
$$

Let $\theta^{*}=F^{-1}\left(t^{*}\right)$ be the type corresponding to the quantile $t^{*}$ and let $q(t)$ be the interim allocation probability corresponding to the allocation rule that assigns a unit to an agent if and only if her type is above $\theta^{*}$ and at most $m-1$ other agents have a higher valuation, i.e.

$$
q(t)=\mathbf{1}_{\left\{t \geq t^{*}\right\}} \phi(t)
$$

We now argue that $q$ majorizes $q^{*}$. By Condition (12) of Proposition 3 we have that for all $t \in[0,1]$

$$
\int_{t}^{1} q^{*}(z) d z \leq \int_{t}^{1} \phi(z) d z
$$

By the definition of the critical quantile $t^{*}$ we obtain that

$$
\int_{t}^{1} q^{*}(z) d z \leq \int_{0}^{1} q^{*}(z) d z=\int_{t^{*}}^{1} \phi(z) d z
$$

Combining, (23) and (24) that for all $t \in[0,1]$

$$
\int_{t}^{1} q^{*}(z) d z \leq \min \left\{\int_{t}^{1} \phi(z) d z, \int_{t^{*}}^{1} \phi(z) d z\right\}=\int_{t}^{1} \mathbf{1}_{t \geq t^{*}} \phi(z) d z=\int_{t}^{1} q(z) d z .
$$

As $\int_{0}^{1} q(z) d z=\int_{0}^{1} q^{*}(z) d z$ by the definitions of $t^{*}$ and $q$, we obtain that $q$ majorizes $q^{*}$. Note that, as the environment is CSM, Lemma 4 implies that $G$ satisfies the conditions of 
Proposition 4. By the Fan-Lorentz Theorem (Proposition 4) we thus have that

$$
\int_{0}^{1} G\left(q^{*}(t), t\right) d t \leq \int_{0}^{1} G(q(t), t) d t
$$

But, as $q^{*}$ is a revenue maximizing interim probability, the above equation must hold with equality. Consequently, every mechanism which implements the interim probability $q(t)=$ $\mathbf{1}_{\left\{t \geq t^{*}\right\}} \phi(t)$ is revenue maximizing.

Finally, observe that, by Lemma $4, G(0, \cdot)=0$. Thus, the expected revenue as a function of $t^{*}$ is given by

$$
t^{*} \mapsto n \int_{0}^{1} G\left(\mathbf{1}_{\left\{t \geq t^{*}\right\}} \phi(t), t\right) d t=n \int_{t^{*}}^{1} G(\phi(t), t) d t
$$

We show now that $\theta \mapsto H(\psi(\theta), \theta)$ changes sign at most once, from negative to positive. Assume that it crosses zero at $\theta^{*} \neq \underline{\theta}$, that is $H\left(\psi\left(\theta^{*}\right), \theta^{*}\right)=0$. Then for any $\theta>\theta^{*}$ we have

$$
H(\psi(\theta), \theta)-H\left(\psi\left(\theta^{*}\right), \theta^{*}\right)=H(\psi(\theta), \theta)-H\left(\psi(\theta), \theta^{*}\right)+H\left(\psi(\theta), \theta^{*}\right)-H\left(\psi\left(\theta^{*}\right), \theta^{*}\right) \geq 0
$$

where the last inequality follows from: (a) the convexity of $H$ which implies that

$$
\frac{H\left(\psi(\theta), \theta^{*}\right)}{\psi(\theta)} \geq \frac{H\left(\psi\left(\theta^{*}\right), \theta^{*}\right)}{\psi\left(\theta^{*}\right)}=0
$$

and hence that

$$
H\left(\psi(\theta), \theta^{*}\right)-H\left(\psi\left(\theta^{*}\right), \theta^{*}\right) \geq 0 .
$$

and (b) the super-modularity of $H$ which implies that

$$
\begin{aligned}
H(\psi(\theta), \theta)-H(0, \theta) & \geq H\left(\psi(\theta), \theta^{*}\right)-H\left(0, \theta^{*}\right) \Longleftrightarrow \\
H(\psi(\theta), \theta)-H\left(\psi(\theta), \theta^{*}\right) & \geq H(0, \theta)-H\left(0, \theta^{*}\right)=0 .
\end{aligned}
$$

Since $F$ is a monotone transformation and since $\theta \mapsto H(\psi(\theta), \theta)$ changes sign at most once, from negative to positive, so does $t \mapsto G(\phi(t), t)$. Since $t \mapsto G(\phi(t), t)$ changes its sign at most once, from negative to positive, (25) this function is quasi-concave, and thus the optimal quantile $t^{*}$ satisfies the first order condition $G\left(\phi\left(t^{*}\right), t^{*}\right)=0$. The result follows because

$$
G\left(\phi\left(t^{*}\right), t^{*}\right)=H\left(\phi\left(t^{*}\right), F^{-1}\left(t^{*}\right)\right)=H\left((\phi \circ F)\left(\theta^{*}\right), \theta^{*}\right)=H\left(\psi_{m, n}\left(\theta^{*}\right), \theta^{*}\right) .
$$


This completes the proof of Theorem 1 .

Proof of Proposition 6: Let $\psi, \psi^{\prime}:(0,1) \rightarrow(0, \infty)$ be continuous functions with $\psi(q) \leq$ $\psi^{\prime}(q)$ for all $q \in[0,1]$. Recall that $\theta \mapsto H(\psi(\theta), \theta)$, and $\theta \mapsto H\left(\psi^{\prime}(\theta), \theta\right)$ change sign only once $^{38}$, from negative to positive, and define $\theta^{*}, \theta^{* *}$ implicitly by

$$
0=H\left(\psi\left(\theta^{*}\right), \theta^{*}\right) \text { and } 0=H\left(\psi^{\prime}\left(\theta^{* *}\right), \theta^{* *}\right)
$$

We have that

$$
0=H\left(\psi\left(\theta^{*}\right), \theta^{*}\right)=\frac{H\left(\psi\left(\theta^{*}\right), \theta^{*}\right)}{\psi\left(\theta^{*}\right)} \leq \frac{H\left(\psi^{\prime}\left(\theta^{*}\right), \theta^{*}\right)}{\psi^{\prime}\left(\theta^{*}\right)},
$$

where the last inequality follows from the convexity of $H$, and from the fact that $H(0, \cdot)=0$. The last inequality implies that $0 \leq H\left(\psi^{\prime}\left(\theta^{*}\right), \theta^{*}\right)$ and, since $\theta \mapsto H\left(\psi^{\prime}(\theta), \theta\right)$ changes sign only once, it follows that $\theta^{*} \geq \theta^{* *}$. As $\phi_{m, n}$ decreases in $n$ and increases in $m$ point-wise, the result follows by setting $\psi=\phi_{m, n} \circ F$.

For the proof of Theorem 2, we first need two Lemmas. It follows from standard arguments that the expected revenue in a mechanism inducing the interim probabilities $\left(p_{1}, p_{2}\right)$ and such that participation constraint for the lowest type of each agent binds is given by

$$
\int_{\underline{\theta}}^{\bar{\theta}} H\left(p_{1}\left(\theta_{1}\right), \theta_{1}\right) d F\left(\theta_{1}\right)+\int_{\underline{\theta}}^{\bar{\theta}} H\left(p_{2}\left(\theta_{2}\right), \theta_{2}\right) d F\left(\theta_{2}\right)
$$

Lemma 5. Fix any optimal $\left(p_{1}^{*}, p_{2}^{*}\right)$ and let $\theta_{i}^{*}=\inf \left\{\theta_{i} \in[\underline{\theta}, \bar{\theta}] \mid p_{i}^{*}\left(\theta_{i}\right)>0\right\}$ be the cutoff type for bidder $i, i=1,2$. Then:

(1) $H\left(p_{i}^{*}\left(\theta_{i}^{*}\right), \theta_{i}^{*}\right) \geq 0$

(2) $p_{1}^{*}\left(\theta_{1}^{*}\right) \geq F\left(\theta_{2}^{*}\right)$ and $p_{2}^{*}\left(\theta_{2}^{*}\right) \geq F\left(\theta_{1}^{*}\right)$.

Proof: (1) This follows by similar arguments to those in the proof for Theorem 1, since otherwise we can improve the mechanism by setting $p_{i}\left(\theta_{i}\right)$ to be zero below $\theta_{i}^{*}$.

(2) Again, by using similar arguments to those in the proof for Theorem 1, we can show

$$
H\left(p_{i}^{*}\left(\theta_{i}\right), \theta_{i}\right)>0
$$

for any $\theta_{i}>\theta_{i}^{*}$. Suppose (2) does not hold. Without loss of generality, we can assume $p_{1}\left(\theta_{1}^{*}\right)<F\left(\theta_{2}^{*}\right)$. Then, there exists some $\tilde{\theta}_{1}>\theta_{1}^{*}$ such that $p_{1}^{*}\left(\theta_{1}\right)<F\left(\theta_{2}^{*}\right)$ for any $\theta_{1}^{*}<\theta_{1} \leq$

\footnotetext{
${ }^{38}$ If $H(\psi(\theta), \theta)$ and $H\left(\psi^{\prime}(\theta), \theta\right)$ do not change sign, define $\theta^{*}$ and $\theta^{* *}$ to be the relevant boundary of the interval $[\underline{\theta}, \bar{\theta}]$.
} 
$\tilde{\theta}_{1}$. Let

$$
\theta_{1}^{\max }=\sup \left\{\theta_{1}^{\prime} \in[\underline{\theta}, \bar{\theta}] \mid p_{1}^{*}\left(\theta_{1}\right)<F\left(\theta_{2}^{*}\right)\right\}
$$

We construct another pair of feasible and incentive compatible interim allocation probabilities $\left(\bar{p}_{1}, \bar{p}_{2}\right)$, such that $\bar{p}\left(\theta_{1}\right)=F\left(\theta_{2}^{*}\right)$ for all $\theta_{1}^{*} \leq \theta_{1} \leq \theta_{1}^{\max }$ and such that $\bar{p}_{1}\left(\theta_{1}\right)=p_{1}^{*}\left(\theta_{1}\right)$ otherwise, and $\bar{p}_{2}\left(\theta_{2}\right)=p_{2}\left(\theta_{2}\right)$ for all $\theta_{2} \in[\underline{\theta}, \bar{\theta}]$.

By convexity of $H$ and by

$$
H\left(p_{i}^{*}\left(\theta_{i}\right), \theta_{i}\right)>0
$$

for any $\theta_{i}>\theta_{i}^{*}$, we obtain

$$
H\left(\bar{p}_{1}\left(\theta_{1}\right), \theta_{1}\right) \geq H\left(p_{1}^{*}\left(\theta_{1}\right), \theta_{1}\right)
$$

for all $\theta_{1} \geq \theta_{1}^{*}$, with strict inequality on some set of positive measure. ${ }^{39}$ Thus $\left(p_{1}^{*}, p_{2}^{*}\right)$ is not optimal, contradicting the earlier assumption.

Lemma 6. Fix any incentive compatible and feasible pair $\left(p_{1}, p_{2}\right)$, and define $\theta_{i}^{0}=\inf \left\{\theta_{i} \in\right.$ $\left.[\underline{\theta}, \bar{\theta}] \mid p_{i}\left(\theta_{i}\right)>0\right\}, i=1,2$. The function $\eta:[\underline{\theta}, \bar{\theta}] \rightarrow[\underline{\theta}, \bar{\theta}]$ defined by

$$
\eta\left(\theta_{1}\right)=F^{-1}\left(p_{1}\left(\theta_{1}\right)\right)
$$

is non-decreasing. Moreover, $p_{2}$ is weakly majorized by

$$
\widetilde{p}_{2}\left(\theta_{2}\right)= \begin{cases}F\left(\eta^{-1}\left(\theta_{2}\right)\right) & \text { if } \theta_{2} \geq \theta_{2}^{0} \\ 0 & \text { otherwise }\end{cases}
$$

where

$$
\eta^{-1}\left(\theta_{2}\right) \equiv\left\{\begin{array}{ll}
\inf \left\{t \in[\underline{\theta}, \bar{\theta}] \mid \eta(t) \geq \theta_{2}\right\} & \text { if } \eta(\bar{\theta}) \geq \theta_{2} \\
\bar{\theta} & \text { otherwise }
\end{array} .\right.
$$

The proof of Lemma 6 crucially relies on the following result by Mitroi and Niculescu (2011, P4. Lemma 3).

Lemma 7 (Young's inequality for non-decreasing functions). Let $\eta:[\underline{\theta}, \bar{\theta}] \rightarrow[\underline{\theta}, \bar{\theta}]$ be a nondecreasing function and let $\eta^{-1}$ be its generalized inverse. Then, for every Lebesgue locally integrable function $K:[0, \infty) \times[0, \infty) \rightarrow[0, \infty)$ and for every pair of nonnegative numbers

\footnotetext{
${ }^{39} \mathrm{As} H$ is convex in $p$ it follows that $p \mapsto \frac{H(p, \theta)-H(0, \theta)}{p}=\frac{H(p, \theta)}{p}$ is non-decreasing, which implies that $p \mapsto H(p, \theta)$ is increasing whenever $H(p, \theta)>0$.
} 
$A<B$,

$$
\int_{A}^{B}\left(\int_{\eta(A)}^{\eta(s)} K(s, w) d w\right) d s+\int_{\eta(A)}^{\eta(B)}\left(\int_{A}^{\eta^{-1}(w)} K(s, w) d s\right) d w=\int_{A}^{B}\left(\int_{\eta(A)}^{\eta(B)} K(s, w) d w\right) d s .
$$

Proof of Lemma 6: The function $\eta=F^{-1} \circ p_{1}$ and its generalized inverse $\eta^{-1}$ are well defined and non-decreasing since $p_{1}$ is non-decreasing and since $F^{-1}$ is well defined (as $F$ admits a strictly positive density).

Fix a feasible pair $\left(p_{1}, p_{2}\right)$. It is without loss of generality to set $\eta(\bar{\theta})=\bar{\theta}$ (i.e. $p_{1}(\bar{\theta})=1$ ) and $\eta(\underline{\theta})=\underline{\theta}$ (i.e. $p_{1}(\underline{\theta})=0$ ), as this affects $p$ only on a set of measure zero. The relevant feasibility constraint (see, for example, Maskin and Riley, 1984) requires that, for any $t \in$ $\left[\theta_{2}^{0}, \bar{\theta}\right]$

$$
\int_{\eta^{-1}(t)}^{\bar{\theta}} p_{1}\left(\theta_{1}\right) d F\left(\theta_{1}\right)+\int_{t}^{\bar{\theta}} p_{2}\left(\theta_{2}\right) d F\left(\theta_{2}\right) \leq 1-F(t) F\left(\eta^{-1}(t)\right) .
$$

Take $A=\eta^{-1}(\underline{\theta})=\underline{\theta}, B=\eta^{-1}(t)$ for any $t \in\left[\theta_{2}^{0}, \bar{\theta}\right]$, and $K(s, w)=f(s) f(w)$. Lemma 7 implies

$$
\begin{gathered}
\int_{\eta^{-1}(\underline{\theta})}^{\eta^{-1}(t)}\left(f(s) \int_{\underline{\theta}}^{\eta(s)} f(w) d w\right) d s+\int_{\underline{\theta}}^{t}\left(f(w) \int_{\underline{\theta}}^{\eta^{-1}(w)} f(s) d s\right) d w=\int_{\eta^{-1}(\underline{\theta})}^{\eta^{-1}(t)}\left(f(s) \int_{\underline{\theta}}^{t} f(w) d w\right) d s \\
\Rightarrow \int_{\underline{\theta}}^{\eta^{-1}(t)} F(\eta(s)) d F(s)+\int_{\underline{\theta}}^{t} F\left(\eta^{-1}(w)\right) d F(w)=F(t) F\left(\eta^{-1}(t)\right)
\end{gathered}
$$

On the other hand, take $A=\underline{\theta}, B=\bar{\theta}, K(s, w)=f(s) f(w)$ and use the earlier assumption that $\eta(\bar{\theta})=\bar{\theta}$ and $\eta(\underline{\theta})=\underline{\theta}$, Lemma 7 yields

$$
\begin{gathered}
\int_{\underline{\theta}}^{\bar{\theta}}\left(f(s) \int_{\eta(\underline{\theta})}^{\eta(s)} f(w) d w\right) d s+\int_{\eta(\underline{\theta})}^{\eta(\bar{\theta})}\left(f(w) \int_{\underline{\theta}}^{\eta^{-1}(w)} f(s) d s\right) d w=\int_{\underline{\theta}}^{\bar{\theta}}\left(f(w) \int_{\eta(\underline{\theta})}^{\eta(\bar{\theta})} f(s) d s\right) d w \\
\Rightarrow \int_{\underline{\theta}}^{\bar{\theta}} F(\eta(\theta)) d F(\theta)+\int_{\underline{\theta}}^{\bar{\theta}} F\left(\eta^{-1}(\theta)\right) d F(\theta)=1
\end{gathered}
$$

Taking the difference between equations (24) and (25) further yields

$$
\int_{\eta^{-1}(t)}^{\bar{\theta}} F\left(\eta\left(\theta_{1}\right)\right) d F\left(\theta_{1}\right)+\int_{t}^{\bar{\theta}} F\left(\eta^{-1}\left(\theta_{2}\right)\right) d F\left(\theta_{2}\right)=1-F(t) F\left(\eta^{-1}(t)\right) .
$$


As $p_{1}=F \circ \eta$, inequality (26) and equation (29) imply together that, for any $t \geq \eta\left(\theta_{1}^{0}\right)$,

$$
\int_{t}^{\bar{\theta}} p_{2}\left(\theta_{2}\right) d F\left(\theta_{2}\right) \leq \int_{t}^{\bar{\theta}} F\left(\eta^{-1}\left(\theta_{2}\right)\right) d F\left(\theta_{2}\right)
$$

i.e. $\widetilde{p}_{2}\left(\theta_{2}\right)=F\left(\eta^{-1}\left(\theta_{2}\right)\right)$ weakly majorizes any feasible $p_{2}$.

Proof of Theorem 2: Fix any optimal $\left(p_{1}^{*}, p_{2}^{*}\right)$ with cutoff types $\left(\theta_{1}^{*}, \theta_{2}^{*}\right)$ defined by

$$
\theta_{i}^{*}=\inf \left\{\theta: p_{i}(\theta)>0\right\}
$$

Lemma 6 implies that $p_{2}^{*}$ is weakly majorized by

$$
\widetilde{p}_{2}\left(\theta_{2}\right)= \begin{cases}F\left(\eta^{-1}\left(\theta_{2}\right)\right) & \text { if } \theta_{2} \geq \theta_{2}^{*} \\ 0 & \text { otherwise }\end{cases}
$$

where $\eta^{-1}$ is defined in Lemma 6 . We now show that $p_{2}^{*}$ is actually majorized by $\widetilde{p}_{2}\left(\theta_{2}\right)$. Suppose not, that is

$$
\int_{\theta_{2}^{*}}^{\bar{\theta}} p_{2}^{*}\left(\theta_{2}\right) d F\left(\theta_{2}\right)<\int_{\theta_{2}^{*}}^{\bar{\theta}} \widetilde{p}_{2}\left(\theta_{2}\right) d F\left(\theta_{2}\right) .
$$

Then, there exists another feasible $p_{2}$, such that: (1) $p_{2}\left(\theta_{2}\right)=0$ for $\theta_{2}<\theta_{2}^{*}$ and (2) $p_{2}\left(\theta_{2}\right) \geq$ $p_{2}^{*}\left(\theta_{2}\right)$ for any $\theta_{2} \geq \theta_{2}^{*}$ with strict inequality on some set of positive measure. By the convexity of $H$ and since

$$
H\left(p_{2}^{*}\left(\theta_{2}\right), \theta_{2}\right)>0
$$

for any $\theta_{2}>\theta_{2}^{*}$ (see Lemma 5), we obtain

$$
H\left(p_{2}\left(\theta_{2}\right), \theta_{2}\right)>H\left(p_{2}^{*}\left(\theta_{2}\right), \theta_{2}\right)
$$

for any $\theta_{2} \geq \theta_{2}^{*}$ with strict inequality on some set of positive measure. Thus, $p_{2}^{*}$ is not optimal, contradicting the earlier assumption, and any optimal $p_{2}^{*}$ must be majorized by $\widetilde{p}_{2}\left(\theta_{2}\right)$ as desired.

We can now apply the Fan-Lorentz Theorem to the second element of the revenue

$$
\int_{\underline{\theta}}^{\bar{\theta}} H\left(p_{1}\left(\theta_{1}\right), \theta_{1}\right) d F\left(\theta_{1}\right)+\int_{\underline{\theta}}^{\bar{\theta}} H\left(p_{2}\left(\theta_{2}\right), \theta_{2}\right) d F\left(\theta_{2}\right) .
$$

(seen as a functional in $p_{2}$ ) and conclude that $\widetilde{p}_{2}$ is optimal. As $p_{1}$ and $\tilde{p}_{2}$ are completely 
determined by $\eta$, we can obtain an upper bound on the seller's expected revenue as a function of $\eta$. It is without loss of generality to set $\eta(\bar{\theta})=\bar{\theta}$ (as this affects $\eta$ only on a set of measure zero). The bound on expected revenue is given by

$$
\begin{aligned}
& \int_{\theta_{1}^{*}}^{\bar{\theta}} H\left(p_{1}\left(\theta_{1}\right), \theta_{1}\right) d F\left(\theta_{1}\right)+\int_{\theta_{2}^{*}}^{\bar{\theta}} H\left(p_{2}\left(\theta_{2}\right), \theta_{2}\right) d F\left(\theta_{2}\right) \\
& \leq \int_{\theta_{1}^{*}}^{\bar{\theta}} H\left(F\left(\eta\left(\theta_{1}\right)\right), \theta_{1}\right) d F\left(\theta_{1}\right)+\int_{\eta\left(\theta_{1}^{*}\right)}^{\eta(\bar{\theta})} H\left(F\left(\eta^{-1}\left(\theta_{2}\right)\right), \theta_{2}\right) d F\left(\theta_{2}\right)
\end{aligned}
$$

where we used $\theta_{2}^{*} \geq \eta\left(\theta_{1}^{*}\right)$ (Lemma $5(2)$ ) to obtain the inequality. To show the optimal mechanism is symmetric, it suffices to show that, optimally, $\eta(\theta)=\theta$ for any $\theta>\theta_{1}^{*}$.

Note that, as $p_{1}^{*}$ is by assumption piecewise differentiable with finitely many jumps, $\eta^{*}$ is also piecewise differentiable with finitely many jumps. Fixing any optimal $\eta^{*}$ there are two possibilities:

(1) There exists an interval $[x, y], x>\theta_{1}^{*}$, on which $\eta^{*}$ does not jump and $\eta^{*}$ is strictly increasing, i.e. $\eta^{* \prime}(\theta)>0$. Then we have

$$
\begin{aligned}
\int_{x}^{y} H\left(F\left(\eta^{*}\left(\theta_{1}\right)\right), \theta_{1}\right) d F\left(\theta_{1}\right)+\int_{\eta^{*}(x)}^{\eta^{*}(y)} H\left(F\left(\eta^{*-1}\left(\theta_{2}\right)\right), \theta_{2}\right) d F\left(\theta_{2}\right) \\
=\int_{x}^{y} H\left(F\left(\eta^{*}(\theta)\right), \theta\right) d F(\theta)+\int_{x}^{y} H\left(F(\theta), \eta^{*}(\theta)\right) d F\left(\eta^{*}(\theta)\right) \\
=\int_{x}^{y}\left[H\left(F\left(\eta^{*}(\theta)\right), \theta\right) f(\theta)+H\left(F(\theta), \eta^{*}(\theta)\right) f\left(\eta^{*}(\theta)\right) \eta^{* \prime}(\theta)\right] d \theta .
\end{aligned}
$$

Define

$$
\hat{H}\left(\theta, \eta, \eta^{\prime}\right) \equiv H(F(\eta(\theta)), \theta) f(\theta)+H(F(\theta), \eta(\theta)) f(\eta(\theta)) \eta^{\prime}(\theta) .
$$

Since $\eta^{*}(\theta)$ is optimal, it must be the solution to the following calculus of variation problem.

$$
\max _{\eta} I(\eta) \equiv \int_{x}^{y} \hat{H}\left(\theta, \eta, \eta^{\prime}\right) d \theta
$$

subject to the inequality constraint $\eta^{* \prime}(\theta) \geq 0$, and subject to the boundary conditions $\eta(x)=\eta^{*}(x)$ and $\eta(y)=\eta^{*}(y)$.

This is a relatively standard calculus of variation problem (see, for example, Sagan (1992), Chapter 2.3). Since the constraint is not binding anywhere on $(x, y)$, a necessary condition for $\eta^{*}(\theta)$ being optimal is that it solves the Euler-Lagrange condition almost everywhere on 
$(x, y)$. The Euler-Lagrange condition requires that, for all $\theta$,

$$
\frac{\partial \hat{H}\left(\theta, \eta(\theta), \eta^{\prime}(\theta)\right)}{\partial \eta}-\frac{d}{d \theta} \frac{\partial \hat{H}\left(\theta, \eta(\theta), \eta^{\prime}(\theta)\right)}{\partial \eta^{\prime}}=0
$$

where

$$
\begin{aligned}
\frac{\partial \hat{H}}{\partial \eta}=\frac{\partial H}{\partial p}(F(\eta(\theta)), \theta) f(\eta(\theta)) f(\theta)+\frac{\partial H}{\partial \theta}(F(\theta), & \left.\eta(\theta)) f(\eta(\theta)) \eta^{\prime}(\theta)\right) \\
& +f^{\prime}(\eta(\theta)) H(F(\theta), \eta(\theta)) \eta^{\prime}(\theta)
\end{aligned}
$$

and where

$$
\begin{aligned}
\frac{d}{d \theta} \frac{\partial \hat{H}}{\partial \eta^{\prime}} & =\frac{d}{d \theta} H(F(\theta), \eta(\theta)) f(\eta(\theta)) \\
& \left.=\frac{\partial H}{\partial p}(F(\theta), \eta(\theta)) f(\theta) f(\eta(\theta))+\frac{\partial H}{\partial \theta}(F(\theta), \eta(\theta)) f(\eta(\theta)) \eta^{\prime}(\theta)\right)+ \\
& f^{\prime}(\eta(\theta)) H(F(\theta), \eta(\theta)) \eta^{\prime}(\theta) .
\end{aligned}
$$

Thus, we obtain the following necessary condition:

$$
\begin{aligned}
\frac{\partial \hat{H}\left(\theta, \eta(\theta), \eta^{\prime}(\theta)\right)}{\partial \eta}-\frac{d}{d \theta} \frac{\partial \hat{H}\left(\theta, \eta(\theta), \eta^{\prime}(\theta)\right)}{\partial \eta^{\prime}} & =0 \\
\Leftrightarrow \frac{\partial H}{\partial p}(F(\eta(\theta)), \theta) & =\frac{\partial H}{\partial p}(F(\theta), \eta(\theta)) .
\end{aligned}
$$

$\eta(\theta)=\theta$ is the unique solution of the above Euler-Lagrange equation since we assumed

$$
f(\theta) \frac{\partial^{2} H}{\partial^{2} p}(F(\theta), \theta)<\frac{\partial^{2} H}{\partial p \partial \theta}(F(\theta), \theta)
$$

for any $\theta$ in $[\underline{\theta}, \bar{\theta}]$, which implies that the derivative of $\frac{\partial H}{\partial p}(F(\eta(\theta)), \theta)$ with respect to $\eta$ is always smaller that the derivative of $\frac{\partial H}{\partial p}(F(\theta), \eta(\theta))$ with respect to $\eta$. This establishes that on any interval $[x, y]$ where $\eta^{*}$ is continuous and strictly increasing, $\eta^{*}$ is the identity.

(2) There exists an interval $\left[x^{\prime}, y^{\prime}\right)$ such that $\eta^{*}$ is constant on that interval, i.e. $\eta^{*}(\theta)=k^{*}$ for any $\theta \in\left[x^{\prime}, y^{\prime}\right)$. We prove that this possibility cannot arise in 2 steps:

Step 1: We show that either $\eta^{*}\left(x^{\prime-}\right)=k^{*}$ or $\eta^{*}\left(y^{\prime+}\right)=k^{*}$. For any $k \in\left(\eta\left(x^{\prime-}\right), \eta\left(y^{\prime+}\right)\right)$, consider the non-decreasing function $\eta^{k}(\theta)$ such that $\eta^{k}(\theta)=k$ for any $\theta \in\left(x^{\prime}, y^{\prime}\right)$ and 
$\eta^{k}(\theta)=\eta^{*}(\theta)$ otherwise. If the seller uses $\eta^{k}$, then her revenue can be written as

$$
\begin{array}{rl}
\int_{x^{\prime}}^{y^{\prime}} H & H\left(F\left(\eta^{k}\left(\theta_{1}\right)\right), \theta_{1}\right) d F\left(\theta_{1}\right)+\int_{\eta\left(x^{\prime-}\right)}^{\eta\left(y^{\prime+}\right)} H\left(F\left(\left(\eta^{k}\right)^{-1}\left(\theta_{2}\right)\right), \theta_{2}\right) d F\left(\theta_{2}\right)+M \\
=\int_{x^{\prime}}^{y^{\prime}} H(F(k), \theta) d F(\theta)+\int_{\eta^{*}\left(x^{\prime}\right)}^{k} H\left(F\left(x^{\prime}\right), \theta\right) d F(\theta)+\int_{k}^{\eta^{*}\left(y^{\prime+}\right)} H\left(F\left(y^{\prime}\right), \theta\right) d F(\theta)+M
\end{array}
$$

where $M$ is some term independent of $k$. Taking the derivative of the above expression with respect to $k$ yields

$$
\begin{aligned}
f(k) \int_{x^{\prime}}^{y^{\prime}} \frac{\partial H}{\partial p}(F(k), \theta) d F(\theta)+f(k)\left[H\left(F\left(x^{\prime}\right), k\right)-H\left(F\left(y^{\prime}\right), k\right)\right] \\
=f(k)\left\{\int_{x^{\prime}}^{y^{\prime}}\left[\frac{\partial H}{\partial p}(F(k), \theta)-\frac{\partial H}{\partial p}(F(\theta), k)\right] d F(\theta)\right\} .
\end{aligned}
$$

A necessary condition for $\eta^{*}(\theta)=\eta^{k^{*}}(\theta)$ being optimal is

$$
\int_{x^{\prime}}^{y^{\prime}}\left[\frac{\partial H}{\partial p}\left(F\left(k^{*}\right), \theta\right)-\frac{\partial H}{\partial p}\left(F(\theta), k^{*}\right)\right] d F(\theta)=0 .
$$

Observe that

$$
\frac{\partial H}{\partial p}\left(F\left(k^{*}\right), \theta\right)-\frac{\partial H}{\partial p}\left(F(\theta), k^{*}\right)
$$

equals 0 at $\theta=k^{*}$ and is increasing in $\theta$ as we have assumed $\frac{\partial H}{\partial p \partial \theta}(F(\theta), \theta)>\frac{\partial H}{\partial^{2} p}(F(\theta), \theta) f(\theta)$. Then

$$
\int_{x^{\prime}}^{y^{\prime}}\left[\frac{\partial H}{\partial p}\left(F\left(k^{*}\right), \theta\right)-\frac{\partial H}{\partial p}\left(F(\theta), k^{*}\right)\right] d F(\theta)=0
$$

implies $x^{\prime}<k^{*}<y^{\prime}$ and

$$
\int_{x^{\prime}}^{k^{*}}\left[\frac{\partial H}{\partial p}\left(F\left(k^{*}\right), \theta\right)-\frac{\partial H}{\partial p}\left(F(\theta), k^{*}\right)\right] d F(\theta)<0 .
$$

Then, for any $s \in\left(\max \left\{x^{\prime}, \eta^{*}\left(x^{\prime-}\right)\right\}, k^{*}\right]$, we construct $\eta^{s}$ such that $\eta^{s}(\theta)=s$ for $\theta \in\left(x^{\prime}, k^{*}\right)$ 
and $\eta^{s}(\theta)=\eta^{*}(\theta)$ otherwise. If the seller uses $\eta^{s}$, then her revenue can be written as

$$
\begin{aligned}
& \int_{x^{\prime}}^{k^{*}} H\left(F\left(\eta^{s}\left(\theta_{1}\right)\right), \theta_{1}\right) d F\left(\theta_{1}\right)+\int_{\eta\left(x^{\prime}\right)}^{k^{*}} H\left(F\left(\left(\eta^{s}\right)^{-1}\left(\theta_{2}\right)\right), \theta_{2}\right) d F\left(\theta_{2}\right)+M_{1} \\
= & \int_{x^{\prime}}^{k^{*}} H(F(s), \theta) d F(\theta)+\int_{\eta^{*}\left(x^{\prime}\right)}^{s} H\left(F\left(x^{\prime}\right), \theta\right) d F(\theta)+\int_{s}^{k^{*}} H(F(s), \theta) d F(\theta)+M_{1}
\end{aligned}
$$

where $M_{1}$ is some term independent of $s$. Taking the derivative of the above expression with respect to $s$ yields

$$
f(s) \int_{x^{\prime}}^{k^{*}}\left[\frac{\partial H}{\partial p}(F(s), \theta)-\frac{\partial H}{\partial p}(F(\theta), s)\right] d F(\theta) .
$$

Recall that we have shown

$$
\int_{x^{\prime}}^{k^{*}}\left[\frac{\partial H}{\partial p}\left(F\left(k^{*}\right), \theta\right)-\frac{\partial H}{\partial p}\left(F(\theta), k^{*}\right)\right] d F(\theta)<0,
$$

and hence there exists $\varepsilon>0$ such that

$$
\int_{x^{\prime}}^{k^{*}}\left[\frac{\partial H}{\partial p}(F(s), \theta)-\frac{\partial H}{\partial p}(F(\theta), s)\right] d F(\theta)<0
$$

for any $s \in\left(k^{*}-\varepsilon, k^{*}\right)$. Thus $\eta^{k^{*}-\varepsilon}$ is strictly more profitable to the seller than $\eta^{*}=\eta^{k^{*}}$, contradicting the assumption that $\eta^{*}$ is optimal. Hence, we have shown that if $\eta^{*} \equiv k^{*}$ is constant on an interval $\left[x^{\prime}, y^{\prime}\right)$ it must be that $\eta^{*}$ is either left-continuous at $x^{\prime}$ or rightcontinuous at $y^{\prime}$. Below we assume that $\eta^{*}$ is left-continuous at $x^{\prime}$ - the proof for the other case is essentially the same.

Step 2: Suppose there exists an interval $\left[x^{\prime}, y^{\prime}\right)$ such that $\eta^{*}(\theta)=k^{*}$ if and only if $\theta \in\left[x^{\prime}, y^{\prime}\right)$. By assumption, there must exist $z^{\prime}<x^{\prime}$ such that $\eta^{*}$ is strictly increasing on $\left(z^{\prime}, x^{\prime}\right)$. Then Step 1 and case (1) together imply $k^{*}=\eta^{*}\left(x^{-}\right)=x^{\prime}$. It also follows that $\eta^{*}\left(y^{\prime}\right)>k^{*}$. For any $r \in\left[x^{\prime}, y^{\prime}\right]$, we construct $\eta^{r}$ such that $\eta^{r}(\theta)=\min \left\{y^{\prime}, \eta^{*}\left(y^{\prime}\right)\right\}$ on $\left[r, y^{\prime}\right)$ and $\eta^{r}(\theta)=\eta^{*}(\theta)$ otherwise. By construction, if $\eta^{*}$ is non-decreasing, then $\eta^{r}$ is also non-decreasing. 
If the seller uses $\eta^{r}$ instead of $\eta^{*}$, the change in the expected revenue equals

$$
\begin{aligned}
\triangle(r)= & \int_{r}^{y^{\prime}} H\left(F\left(\min \left\{y^{\prime}, \eta^{*}\left(y^{\prime}\right)\right\}, \theta\right) d F(\theta)+\int_{\eta^{*}\left(x^{\prime}\right)}^{\min \left\{y^{\prime}, \eta^{*}\left(y^{\prime}\right)\right\}} H(F(r), \theta) d F(\theta)\right. \\
& -\int_{r}^{y^{\prime}} H\left(F\left(x^{\prime}\right), \theta\right) d F(\theta)-\int_{\eta^{*}\left(x^{\prime}\right)}^{\min \left(y^{\prime}, \eta^{*}\left(y^{\prime}\right)\right)} H\left(F\left(y^{\prime}\right), \theta\right) d F(\theta)
\end{aligned}
$$

Taking the derivative of the above expression with respect to $r$ yields

$$
\triangle^{\prime}(r)=f(r) \int_{x^{\prime}}^{\min \left\{y^{\prime}, \eta^{*}\left(y^{\prime}\right)\right\}}\left[\frac{\partial H(F(r), \theta)}{\partial p}-\frac{\partial H(F(\theta), r)}{\partial p}\right] d F(\theta)
$$

By similar arguments as used in Step 1, it can be verified that $\triangle\left(y^{\prime}\right)=0$ and $\triangle^{\prime}(r)<0$ for $r=y^{\prime}$. Hence there exists $r \in\left[x, y^{\prime}\right)$ such that $\triangle(r)>0$, and thus that $\eta^{*}$ is not optimal.

By (1) and (2), we now conclude that, if it exists, the unique optimal solution to the revenue maximizing problem is $\eta^{*}(\theta)=\theta$ for any $\theta>\theta_{1}^{*}$.

Proof of Proposition 9: Let $\left(a^{*}, x^{*}\right)$ be the (potentially assymetric) welfare investment and allocation profile, absent any incentive constraints. We show that this profile can be implemented as an equilibrium of the game where agents first chose investments and then participate in an $m+1$-price auction. Clearly, allocating to the agents with the $m$ highest expost valuations $v\left(a_{i}, \theta_{i}\right)$ is welfare-maximizing for every fixed vector of investments strategies $a_{1}, \ldots, a_{n}: \mathbb{R}_{+} \rightarrow A$. Thus, the equilibrium of the $m+1$-price auction where each agents bids their value implements the welfare-maximizing allocation of the object for any investment strategy profile. We are thus left to show that we can support the efficient investments as an equilibrium. Let $x^{*}\left(v_{1}, \ldots, v_{n}\right)$ be the rule that allocates objects to the agents with the $m$-highest ex-post values, and let $x_{-i}^{*}$ the rule that allocates objects to the agents excluding $i$ that have the $m$ highest ex-post values. For type profile $\theta \in \mathbb{R}_{+}^{n}$ and investment profile $a \in A^{n}$, let

$$
W(a, \theta)=\sum_{j \in N} x_{j}^{*}\left(v_{1}\left(a_{1}, \theta_{1}\right), \ldots, v_{n}\left(a_{n}, \theta_{n}\right)\right) v_{j}\left(a_{j}, \theta_{j}\right)
$$

be the ex-post realized welfare from the allocation (excluding the cost of investment!) and let

$$
W_{-i}\left(a_{-i}, \theta_{-i}\right)=\sum_{j \neq i}\left(x_{-i}^{*}\right)_{j}\left(v_{1}\left(a_{1}, \theta_{1}\right), \ldots, v_{n}\left(a_{n}, \theta_{n}\right)\right) v_{j}\left(a_{j}, \theta_{j}\right)
$$

be the ex-post welfare in the situation where the object cannot be allocated to agent $i$.

Recall that $a^{*}$ is the (potentially asymmetric) welfare-maximizing profile of investment 
strategies. If agent $i$ makes an investment $a_{i} \in A$ and all other agents use the welfare maximizing investment strategies $a_{-i}^{*}$, then $i^{\prime}$ s expected utility in the VCG mechanism is given by

$$
\mathbb{E}_{\theta_{-i}}\left[W\left(a_{i}, a_{-i}^{*}\left(\theta_{-i}\right), \theta\right)-W_{-i}\left(a_{-i}^{*}\left(\theta_{-i}\right), \theta_{-i}\right)-c\left(a_{i}\right) \mid \theta_{i}\right]
$$

As both the second summand above and $\sum_{j \neq i} c\left(a_{j}^{*}\left(\theta_{j}\right)\right)$ are independent of $a_{i}$, we obtain that $a_{i}^{*}(\theta)$ is an equilibrium investment strategy if and only if

$$
a_{i}^{*}(\theta) \in \underset{a_{i} \in A}{\arg \max } \mathbb{E}\left[W\left(a_{i}, a_{-i}^{*}\left(\theta_{-i}\right), \theta\right)-c\left(a_{i}\right)-\sum_{j \neq i} c\left(a_{j}^{*}\left(\theta_{j}\right)\right) \mid \theta_{i}\right] .
$$

which is satisfied since $a^{*}$ was chosen to be the welfare-maximizing investment profile.

\section{Online Appendix: Not for Publication}

Proof of Proposition 5: Agent $i$ 's utility in a symmetric equilibrium where all biders but $i$ use strategy $\beta$ and where $i$ submits a bid $b$ is given by

$$
h\left(\psi_{m, n}\left(\beta^{-1}(b)\right), \theta_{i}\right)-\psi_{m, n}\left(\beta^{-1}(b)\right) b
$$

Taking the derivative with respect to $b$ yields that

$$
\left[\frac{\partial h\left(\psi_{m, n}\left(\beta^{-1}(b)\right), \theta_{i}\right)}{\partial p}-b\right] \frac{\partial \psi_{m, n}\left(\beta^{-1}(b)\right)}{\partial \theta} \frac{\partial \beta^{-1}(b)}{\partial b}-\psi_{m, n}\left(\beta^{-1}(b)\right) \text {. }
$$

Plugging $b=\beta(\theta)$ (as it is optimal for agent $i$ to make the equilibrium bid) yields

$$
\left[\frac{\partial h\left(\psi_{m, n}\left(\theta_{i}\right), \theta_{i}\right)}{\partial p}-\beta\left(\theta_{i}\right)\right] \frac{\partial \psi_{m, n}\left(\theta_{i}\right)}{\partial \theta} \frac{1}{\beta^{\prime}\left(\theta_{i}\right)}-\psi_{m, n}\left(\theta_{i}\right)=0 .
$$

Rearranging for $\beta^{\prime}$ yields the following differential equation:

$$
\beta^{\prime}\left(\theta_{i}\right)=\frac{\partial \psi_{m, n}\left(\theta_{i}\right)}{\partial \theta} \frac{1}{\psi_{m, n}\left(\theta_{i}\right)}\left[\frac{\partial h\left(\psi_{m, n}\left(\theta_{i}\right), \theta_{i}\right)}{\partial p}-\beta\left(\theta_{i}\right)\right]
$$

Since $\theta_{i} \mapsto \frac{\partial \psi_{m, n}\left(\theta_{i}\right)}{\partial \theta} \frac{1}{\psi_{m, n}\left(\theta_{i}\right)}$ is continuous and since the right-hand side of (31) is uniformly Lipschitz in $\beta$, the O.D.E (31) admits a unique solution for every initial value. Set $\theta^{\prime} \equiv$ 
$\theta_{m, n, \mathcal{R}}^{\prime}$ to be the solution to the equation

$$
\frac{h\left(\psi_{m, n}\left(\theta^{\prime}\right), \theta^{\prime}\right)}{\psi_{m, n}\left(\theta^{\prime}\right)}=\mathcal{R}
$$

If we set $\beta\left(\theta^{\prime}\right)=\mathcal{R}$ then the agent with type $\theta^{\prime}$ is, by construction, indifferent between bidding $\mathcal{R}$ and bidding zero. For all higher types $\theta \in\left(\theta^{\prime}, \bar{\theta}\right]$ we extend the bidding function by solving the ODE (31). Since (31) is linear, we obtain the explicit solution

$$
\beta\left(\theta_{i}\right)=\mathcal{R} \frac{\psi_{m, n}\left(\theta^{\prime}\right)}{\psi_{m, n}\left(\theta_{i}\right)}+\frac{1}{\psi_{m, n}\left(\theta_{i}\right)} \int_{\theta^{\prime}}^{\theta_{i}} \frac{\partial \psi_{m, n}(z)}{\partial \theta} \frac{\partial h\left(\psi_{m, n}(z), z\right)}{\partial p} d z
$$

To verify, take the derivative to obtain

$$
\begin{aligned}
\beta^{\prime}\left(\theta_{i}\right) & =-\frac{\partial \psi_{m, n}\left(\theta_{i}\right)}{\partial \theta} \frac{\psi_{m, n}\left(\theta^{\prime}\right)}{\left(\psi_{m, n}\left(\theta_{i}\right)\right)^{2}} \mathcal{R}-\frac{\partial \psi_{m, n}\left(\theta_{i}\right)}{\partial \theta} \frac{1}{\left(\psi_{m, n}\left(\theta_{i}\right)\right)^{2}} \int_{\theta^{\prime}}^{\theta_{i}} \frac{\partial \psi_{m, n}(z)}{\partial \theta} \frac{\partial h\left(\psi_{m, n}(z), z\right)}{\partial p} d z \\
& +\frac{\partial \psi_{m, n}\left(\theta_{i}\right)}{\partial \theta} \frac{1}{\psi_{m, n}\left(\theta_{i}\right)} \frac{\partial h\left(\psi_{m, n}\left(\theta_{i}\right), \theta_{i}\right)}{\partial p} \\
& =-\frac{\partial \psi_{m, n}\left(\theta_{i}\right)}{\partial \theta} \frac{\beta\left(\theta_{i}\right)}{\psi_{m, n}\left(\theta_{i}\right)}+\frac{\partial \psi_{m, n}\left(\theta_{i}\right)}{\partial \theta} \frac{1}{\psi_{m, n}\left(\theta_{i}\right)} \frac{\partial h\left(\psi_{m, n}\left(\theta_{i}\right), \theta_{i}\right)}{\partial p} \\
& =\frac{\partial \psi_{m, n}\left(\theta_{i}\right)}{\partial \theta} \frac{1}{\psi_{m, n}\left(\theta_{i}\right)}\left[\frac{\partial h\left(\psi_{m, n}\left(\theta_{i}\right), \theta_{i}\right)}{\partial p}-\beta\left(\theta_{i}\right)\right] .
\end{aligned}
$$

We first need to show that the solution $\beta$ of (31) is increasing for all $\theta_{i} \geq \theta^{\prime}$. Observe that:

$$
\beta^{\prime} \geq 0 \Leftrightarrow \mathcal{R} \psi_{m, n}\left(\theta^{\prime}\right) \frac{1}{\psi_{m, n}\left(\theta_{i}\right)}+\frac{1}{\psi_{m, n}\left(\theta_{i}\right)} \int_{\theta^{\prime}}^{\theta_{i}} \frac{\partial \psi_{m, n}(z)}{\partial \theta} \frac{\partial h\left(\psi_{m, n}(z), z\right)}{\partial p} d z \leq \frac{\partial h\left(\psi_{m, n}\left(\theta_{i}\right), \theta_{i}\right)}{\partial p}
$$

Plugging the expression for $\mathcal{R}$, this becomes:

$$
\frac{h\left(\psi_{m, n}\left(\theta^{\prime}\right), \theta^{\prime}\right)}{\psi_{m, n}\left(\theta_{i}\right)}+\frac{1}{\psi_{m, n}\left(\theta_{i}\right)} \int_{\theta^{\prime}}^{\theta_{i}} \frac{\partial \psi_{m, n}(z)}{\partial \theta} \frac{\partial h\left(\psi_{m, n}(z), z\right)}{\partial p} d z \leq \frac{\partial h\left(\psi_{m, n}\left(\theta_{i}\right), \theta_{i}\right)}{\partial p}
$$


By super-modularity, we can bound the left hand side above:

$$
\begin{aligned}
& \frac{h\left(\psi_{m, n}\left(\theta^{\prime}\right), \theta^{\prime}\right)}{\psi_{m, n}\left(\theta_{i}\right)}+\frac{1}{\psi_{m, n}\left(\theta_{i}\right)} \int_{\theta^{\prime}}^{\theta_{i}} \frac{\partial \psi_{m, n}(z)}{\partial \theta} \frac{\partial h\left(\psi_{m, n}(z), z\right)}{\partial p} d z \\
& \leq \frac{h\left(\psi_{m, n}\left(\theta^{\prime}\right), \theta^{\prime}\right)}{\psi_{m, n}\left(\theta_{i}\right)}+\frac{\frac{\partial h\left(\psi_{m, n}\left(\theta_{i}\right), \theta_{i}\right)}{\partial p}}{\psi_{m, n}\left(\theta_{i}\right)}\left(\psi_{m, n}\left(\theta_{i}\right)-\psi_{m, n}\left(\theta^{\prime}\right)\right. \\
& =\frac{h\left(\psi_{m, n}\left(\theta^{\prime}\right), \theta^{\prime}\right)}{\psi_{m, n}\left(\theta_{i}\right)}+\frac{\partial h\left(\psi_{m, n}\left(\theta_{i}\right), \theta_{i}\right)}{\partial p}-\frac{\psi_{m, n}\left(\theta^{\prime}\right)}{\psi_{m, n}\left(\theta_{i}\right)} \frac{\partial h\left(\psi_{m, n}\left(\theta_{i}\right), \theta_{i}\right)}{\partial p}
\end{aligned}
$$

Thus, we need to show that:

$$
\begin{aligned}
\frac{h\left(\psi_{m, n}\left(\theta^{\prime}\right), \theta^{\prime}\right)}{\psi_{m, n}\left(\theta_{i}\right)}+\frac{\partial h\left(\psi_{m, n}\left(\theta_{i}\right), \theta_{i}\right)}{\partial p}-\frac{\psi_{m, n}\left(\theta^{\prime}\right)}{\psi_{m, n}(\theta)} \frac{\partial h\left(\psi_{m, n}\left(\theta_{i}\right), \theta_{i}\right)}{\partial p} & \leq \frac{\partial h\left(\psi_{m, n}\left(\theta_{i}\right), \theta_{i}\right)}{\partial p} \Longleftrightarrow \\
\frac{1}{\psi_{m, n}\left(\theta_{i}\right)}\left[h\left(\psi_{m, n}\left(\theta^{\prime}\right), \theta^{\prime}\right)-\psi_{m, n}\left(\theta^{\prime}\right) \frac{\partial h\left(\psi_{m, n}\left(\theta_{i}\right), \theta_{i}\right)}{\partial p}\right] & \leq 0 \Longleftrightarrow \\
\frac{h\left(\psi_{m, n}\left(\theta^{\prime}\right), \theta^{\prime}\right)}{\psi_{m, n}\left(\theta^{\prime}\right)} & \leq \frac{\partial h\left(\psi_{m, n}\left(\theta_{i}\right), \theta_{i}\right)}{\partial p}
\end{aligned}
$$

The last inequality holds by the convexity and super-modularity of $h$.

We need to verify that it is indeed optimal for the type $\theta_{i}$ to bid $\beta\left(\theta_{i}\right)$. We start by considering types $\theta_{i} \in\left[\theta^{\prime}, \bar{\theta}\right]$. By construction, the bid $\beta\left(\theta_{i}\right)$ satisfies the agent's first order condition. If the agent deviates by making the equilibrium bid that is optimal for type $\hat{\theta}$, her utility equals

$$
h\left(\psi_{m, n}(\hat{\theta}), \theta_{i}\right)-\psi_{m, n}(\hat{\theta}) \beta(\hat{\theta}) .
$$

The derivative with respect to $\hat{\theta}$ is

$$
\begin{aligned}
& {\left[\frac{\partial h\left(\psi_{m, n}(\hat{\theta}), \theta_{i}\right)}{\partial p}-\beta(\hat{\theta})\right] \frac{\partial \psi_{m, n}(\hat{\theta})}{\partial \theta}-\psi_{m, n}(\hat{\theta}) \beta^{\prime}(\hat{\theta})} \\
& =\underbrace{\left[\frac{\partial h\left(\psi_{m, n}(\hat{\theta}), \hat{\theta}\right)}{\partial p}-\beta(\hat{\theta})\right] \frac{\partial \psi_{m, n}(\hat{\theta})}{\partial \theta}-\psi_{m, n}(\hat{\theta}) \beta^{\prime}(\hat{\theta})}_{\psi_{m, n}(\hat{\theta}) \beta^{\prime}(\hat{\theta})} \\
& =\frac{\partial h\left(\psi_{m, n}(\hat{\theta}), \theta_{i}\right)}{\partial p}-\frac{\partial h\left(\psi_{m, n}(\hat{\theta}), \hat{\theta}\right)}{\partial p} .
\end{aligned}
$$

(at the last step we used the fact that $\beta$ solves the ODE (31). As $h$ is super-modular, 
expression (33) is increasing, and changes its sign from positive to negative at $\theta_{i}=\hat{\theta}$. Thus, the agent's objective (32) is concave, and is maximized at $\theta_{i}=\hat{\theta}$. An agent of type $\theta_{i} \in\left[\theta^{\prime}, \bar{\theta}\right]$ thus prefers to make the bid $\beta\left(\theta_{i}\right)$ over any other bid in $\left[\beta\left(\theta^{\prime}\right), \beta(\overline{(} \theta)\right]=[R, \beta(\bar{\theta})]$. Clearly it can never be optimal for the agent to make a bid higher than $\beta(\bar{\theta})$ as a bid of $\beta(\bar{\theta})$ would already ensure that she wins and pays strictly less. It remains to verify that the agent does not want to make a bid of zero: observe that the agent could deviate to bid $\mathcal{R}$, which would yield a utility higher than the equilibrium utility of the type $\theta^{\prime}$. It thus suffices to verify that the equilibrium utility of the type $\theta^{\prime}$ is non-negative. This type's equilibrium utility is given by

$$
h\left(\psi_{m, n}\left(\theta^{\prime}\right), \theta^{\prime}\right)-\psi_{m, n}\left(\theta^{\prime}\right) \mathcal{R}
$$

that, by the definition of the reserve price, equals zero. Finally, we verify that no type $\theta_{i} \in\left[\underline{\theta}, \theta^{\prime}\right]$ wants to deviate by making a non-zero bid. To see this, note that by deviating to any bid in $[\mathcal{R}, \beta(\bar{\theta})]$ such a type would get a utility which is lower than the utility the type $\theta^{\prime}$ gets from making this bid. But, by construction, the optimal bid of type $\theta^{\prime}$ equals $\mathcal{R}$ and yields her a utility of zero. This implies that the utility resulting from any bid greater zero must be less than zero for all lower types.

Proof of Proposition 7: Proof: We treat here $m$ and $n$ as real variables. As the natural numbers are a subset of the reals, it is clear that the monotonicity properties also hold on the domain of the natural numbers.

Taking the revenue's derivative of (18) with respect to $n$ we get

$$
\begin{gathered}
\frac{\partial \mathcal{R}^{*}}{\partial n}=\frac{\partial v\left(a_{m, n}\left(\theta_{m, n}^{*}\right), \theta_{m, n}^{*}\right)}{\partial \theta} \frac{\partial}{\partial \theta} \frac{1-F\left(\theta_{m, n}^{*}\right)}{f\left(\theta_{m, n}^{*}\right)} \frac{\partial \theta_{m, n}^{*}}{\partial n}+\frac{1-F\left(\theta_{m, n}^{*}\right)}{f\left(\theta_{m, n}^{*}\right)} \frac{\partial^{2} v\left(a_{m, n}\left(\theta_{m, n}^{*}\right), \theta_{m, n}^{*}\right)}{\partial \theta^{2}} \frac{\partial \theta_{m, n}^{*}}{\partial n} \\
+\frac{1-F\left(\theta_{m, n}^{*}\right)}{f\left(\theta_{m, n}^{*}\right)} \frac{\partial^{2} v\left(a_{m, n}\left(\theta_{m, n}^{*}\right), \theta_{m, n}^{*}\right)}{\partial \theta \partial a} \frac{\partial a_{m, n}^{\prime}\left(\theta_{m, n}^{*}\right)}{\partial n} \\
+\frac{1-F\left(\theta_{m, n}^{*}\right)}{f\left(\theta_{m, n}^{*}\right)} \frac{\partial^{2} v\left(a_{m, n}\left(\theta_{m, n}^{*}\right), \theta_{m, n}^{*}\right)}{\partial \theta \partial a} \frac{\partial a_{m, n}\left(\theta_{m, n}^{*}\right)}{\partial \theta} \frac{\partial \theta_{m, n}^{*}}{\partial n} \\
=\frac{\partial v\left(a_{m, n}\left(\theta_{m, n}^{*}\right), \theta_{m, n}^{*}\right)}{\partial \theta} \frac{\partial}{\partial \theta} \frac{1-F\left(\theta_{m, n}^{*}\right)}{f\left(\theta_{m, n}^{*}\right)} \frac{\partial \theta_{m, n}^{*}}{\partial n}+\frac{1-F\left(\theta_{m, n}^{*}\right)}{f\left(\theta_{m, n}^{*}\right)} \frac{\partial^{2} v\left(a_{m, n}\left(\theta_{m, n}^{*}\right), \theta_{m, n}^{*}\right)}{\partial \theta^{2}} \frac{\partial \theta_{m, n}^{*}}{\partial n} \\
+\frac{1-F\left(\theta_{m, n}^{*}\right)}{f\left(\theta_{m, n}^{*}\right)} \frac{\partial^{2} v\left(a_{m, n}\left(\theta_{m, n}^{*}\right), \theta_{m, n}^{*}\right)}{\partial \theta \partial a}\left[\frac{\partial a_{m, n}\left(\theta_{m, n}^{*}\right)}{\partial n}+\frac{\partial a_{m, n}\left(\theta_{m, n}^{*}\right)}{\partial \theta} \frac{\partial \theta_{m, n}^{*}}{\partial n}\right]<0
\end{gathered}
$$

where the last inequality follows from the increasing hazard rate assumption $\left(\frac{\partial}{\partial \theta} \frac{1-F(\theta)}{f(\theta)}<0\right)$, from Proposition 6 which implies $\frac{\partial \theta_{m, n}^{*}}{\partial n}>0$, from the assumed concavity of $v$ in $\theta$, and from 
the assumed monotonicity of $a_{m, n}\left(\theta_{m, n}^{*}\right)$ in $n$. The proof that $\frac{\partial \mathcal{R}^{*}}{\partial m}>0$ is similar.

Proof of Lemma 3: The expected revenue is given by

$$
R=n \int_{\theta^{*}}^{1}(p)^{\frac{l}{l-1}}\left(\frac{\theta}{b}\right)^{\frac{1}{l-1}} K(\theta) f(\theta) d \theta
$$

where $\frac{l-1}{l} \theta^{*}-\frac{1-F\left(\theta^{*}\right)}{f\left(\theta^{*}\right)}=0$ and where $p=F^{n-1}(\theta)$.

The derivative of the expected revenue with respect to $n$ is

$$
\begin{aligned}
& \int_{\theta^{*}}^{1} F^{\frac{(n-1) l}{l-1}}(\theta)\left(\frac{\theta}{b}\right)^{\frac{1}{l-1}} K(\theta) f(\theta) d \theta+n \int_{\theta^{*}}^{1} F^{\frac{(n-1) l}{l-1}}(\theta) \frac{l}{l-1} \ln F(\theta)\left(\frac{\theta}{b}\right)^{\frac{1}{l-1}} K(\theta) f(\theta) d \theta \\
& =\int_{\theta^{*}}^{1} K(\theta)\left(\frac{\theta}{b}\right)^{\frac{1}{l-1}} F^{\frac{(n-1) l}{l-1}}(\theta)\left[1+n \frac{l}{l-1} \ln F(\theta)\right] f(\theta) d \theta
\end{aligned}
$$

This can be written as:

$$
\int_{\theta^{*}}^{1} K(\theta) \frac{1}{F^{\frac{1}{l-1}}(\theta)}\left(\frac{\theta}{b}\right)^{\frac{1}{l-1}} F^{\frac{(n-1) l}{l-1}}(\theta)\left[1+n \frac{l}{l-1} \ln F(\theta)\right] F^{\frac{1}{l-1}}(\theta) f(\theta) d \theta
$$

Because $1+n \frac{l}{l-1} \ln F(\theta)$ changes sign only once as a function of $\theta$, from negative to positive, and by the concavity of $F$ and monotonicity of $K$, the function $K(\theta)\left(\frac{\theta}{F(\theta)}\right)^{\frac{1}{l-1}}$ is positive and increasing. Thus, it is sufficient to show that ${ }^{40}$

$$
\int_{\theta^{*}}^{1} F^{\frac{(n-1) l}{l-1}}(\theta)\left[1+n \frac{l}{l-1} \ln F(\theta)\right] F^{\frac{1}{l-1}}(\theta) f(\theta) d \theta \geq 0 .
$$

Define a distribution $S(\theta)=F^{\frac{l}{l-1}}(\theta)$ and let $s(\theta)=\frac{l}{l-1} F^{\frac{1}{l-1}}(\theta)$ be its density. Using the

\footnotetext{
${ }^{40}$ Assume $k(\cdot)$ changes sign once at $x^{*} \in[a, b]$ from negative to positive and assume that $d(\cdot)$ is positive increasing on the interval $[a, b]$. Then $\int_{a}^{b} k(t) d t \geq 0$ implies

$$
\int_{a}^{b} k(t) d(t) d t=\int_{a}^{x} k(t) d(t) d t+\int_{x}^{b} k(t) d(t) d t \geq d(x) \int_{a}^{x} k(t) d t+d(x) \int_{x}^{b} k(t) d t=d(x) \int_{a}^{b} k(t) d t \geq 0
$$
}


new notation we have

$$
\begin{aligned}
& \int_{\theta^{*}}^{1} S^{n-1}(\theta)[1+n \ln S(\theta)] s(\theta) d \theta=\int_{\theta^{*}}^{1} S^{n-1}(\theta) s(\theta) d \theta+\int_{\theta^{*}}^{1} S^{n-1}(\theta) n \ln S(\theta) s(\theta) d \theta \\
& =\int_{\theta^{*}}^{1} S^{n-1}(\theta) s(\theta) d \theta+\left.S^{n}(\theta) \ln S(\theta)\right|_{\theta=\theta^{*}} ^{1}-\int_{\theta^{*}}^{1} S^{n}(\theta) \frac{s(\theta)}{S(\theta)} d \theta=-S^{n}\left(\theta^{*}\right) \ln S\left(\theta^{*}\right)>0 .
\end{aligned}
$$

\subsection{Sufficient conditions for CSM Environments}

When does our "virtual utility" function

$$
H(p, \theta)=h(p, \theta)-h_{\theta}(p, \theta) \times \frac{1-F(\theta)}{f(\theta)}
$$

satisfies the conditions in the Fan-Lorentz Theorem, i.e. when is the environment convex super-modular? We have

$$
\frac{\partial^{2} H}{(\partial p)^{2}}=\frac{\partial^{2} h}{(\partial p)^{2}}-\frac{\partial^{3} h}{\partial \theta(\partial p)^{2}}\left(\frac{1-F(\theta)}{f(\theta)}\right)
$$

Since $\frac{\partial^{2} h}{(\partial p)^{2}} \geq 0$ by assumption and because $\frac{1-F(\theta)}{f(\theta)} \geq 0$, a sufficient condition for $\frac{\partial^{2} H}{(\partial p)^{2}} \geq 0$ is $\frac{\partial^{3} h}{\partial \theta(\partial p)^{2}} \leq 0$. We also have

$$
\begin{gathered}
\frac{\partial^{2} H}{\partial \theta \partial p}=\frac{\partial^{2} h}{\partial \theta \partial p}-\frac{\partial^{2} h}{\partial \theta \partial p} \frac{d}{d \theta}\left(\frac{1-F(\theta)}{f(\theta)}\right)-\frac{\partial^{3} h}{(\partial \theta)^{2} \partial p}\left(\frac{1-F(\theta)}{f(\theta)}\right)= \\
\frac{\partial^{2} h}{\partial \theta \partial p}\left(1-\frac{d}{d \theta}\left(\frac{1-F(\theta)}{f(\theta)}\right)\right)-\frac{\partial^{3} h}{(\partial \theta)^{2} \partial p}\left(\frac{1-F(\theta)}{f(\theta)}\right) .
\end{gathered}
$$

A sufficient conditions for $\frac{\partial^{2} H}{\partial \theta \partial p} \geq 0$ is an increasing failure rate (IFR), i.e. $\frac{d}{d \theta}\left(\frac{f(\theta)}{1-F(\theta)}\right) \geq 0$ and $\frac{\partial^{3} h}{(\partial \theta)^{2} \partial p} \leq 0$. To conclude sufficient conditions are :

$$
\text { 1. } \frac{\partial^{3} h}{\partial \theta(\partial p)^{2}} \leq 0, \frac{\partial^{3} h}{(\partial \theta)^{2} \partial p} \leq 0
$$

2. $I F R$

These conditions can be further decomposed into standard sufficient conditions on the functions $v$ and $c$. The above derivations can be compared to the classical treatment (see Guesnerie and Laffont (1984) and Fudenberg and Tirole (1991) Section 7.3.2). They only consider a single agent and hence need not consider the resource constraint that is our main concern 
here. Their sufficient conditions are:

$$
\begin{gathered}
1^{\prime} \cdot \frac{\partial^{3} h}{\partial \theta(\partial p)^{2}} \geq 0, \frac{\partial^{3} h}{(\partial \theta)^{2} \partial p} \leq 0 \\
2^{\prime} \cdot \operatorname{IFR}
\end{gathered}
$$

The difference to our conditions is due to their assumption that the valuation is concave in the allocation. Thus, they initially assume $\frac{\partial^{3} h}{(\partial p)^{2}} \leq 0$, and having $\frac{\partial^{3} h}{\partial \theta(\partial p)^{2}} \geq 0$ ensures that their welfare function is concave so that the First Order Approach can be employed. 